ASC Report No. $1 / 2010$

Mapping Properties of Combined Field Helmholtz Boundary Integral Operators

Jens Markus Melenk 


\section{Most recent ASC Reports}

49/2009 Markus Aurada, Jens Markus Melenk, Dirk Praetorius

Mixed Conforming Elements for the Large-Body Limit in Micromagnetics

48/2009 Irene Reichl, Winfried Auzinger, Heinz-Bodo Schmiedmayer, Ewa Weinmüller Reconstructing the Knee Joint Mechanism from Kinematic Data

47/2009 Irena Rachůnková, Svatoslav Staněk, Ewa Weinmüller, Michael Zenz Neumann Problems with Time Singularities

46/2009 Kazuo Aoki, Ansgar Jüngel, Peter A. Markovich

Small Velocity and finite Temperature Variations in Kinetic Relaxation Models

45/2009 Ansgar Jüngel, Jan-Frederik Mennemann

Time-dependent Simulations of Multidimensional Quantum Waveguides Using a Time-Splitting Spectral method

44/2009 Markus Aurada, Michael Ebner, Samuel Ferraz-Leite, Petra Goldonits, Michael Karkulik, Markus Mayr, Dirk Praetorius

HILBERT - A MATLAB Implementation of Adaptive BEM

43/2009 Matthias Langer, Harald Woracek

A Local Inverse Spectral Theorem for Hamilton Systems

42/2009 Ansgar Jüngel

Energy Transport in Semiconductor Devices

41/2009 Ansgar Jüngel

Global Weak Solutions to Compressible Navier-Stokes Equations for Quantum Fluid

40/2009 Markus Aurada, Petra Goldenits, Dirk Praetorius

Convergence of Data Perturbed Adaptive Boundary Element Methods

Institute for Analysis and Scientific Computing

Vienna University of Technology

Wiedner Hauptstraße 8-10

1040 Wien, Austria

E-Mail: admin@asc.tuwien.ac.at

WWW: http://www.asc.tuwien.ac.at

FAX: $\quad$ +43-1-58801-10196

ISBN 978-3-902627-03-2

(C) Alle Rechte vorbehalten. Nachdruck nur mit Genehmigung des Autors.

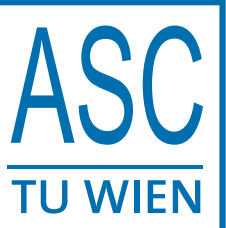




\title{
MAPPING PROPERTIES OF COMBINED FIELD HELMHOLTZ BOUNDARY INTEGRAL OPERATORS
}

\author{
JENS MARKUS MELENK*
}

\begin{abstract}
For the Helmholtz equation (with wavenumber $k$ ) and analytic curves or surfaces $\Gamma$ we analyze the mapping properties of the single layer, double layer as well combined potential boundary integral operators. A $k$-explicit regularity theory for the single layer and double layer potentials is developed, in which these operators are decomposed into three parts: the first part is the single or double layer potential for the Laplace equation, the second part is an operator with finite shift properties, and the third part is an operator that maps into a space of piecewise analytic functions. For all parts, the $k$-dependence is made explicit. We also develop a $k$-explicit regularity theory for the inverse of the combined potential operator $A= \pm 1 / 2+K-\mathbf{i} \eta V$ and its adjoint, where $V$ and $K$ are the single layer and double layer operators for the Helmholtz kernel and $\eta \in \mathbb{R}$ is a coupling parameter with $|\eta| \sim|k|$. The decomposition of the inverses $A^{-1}$ and $\left(A^{\prime}\right)^{-1}$ takes the form of a sum of two operators $A_{1}, A_{2}$ where $A_{1}: H^{s}(\Gamma) \rightarrow H^{s}(\Gamma)$ with bounds independent of $k$ and a smoothing operator $A_{2}$ that maps into a space of analytic functions on $\Gamma$. The $k$-dependence of the mapping properties of $A_{2}$ is made explicit.
\end{abstract}

Key words. high frequency scattering, boundary integral operators, combined field equations, Helmholtz equation, regularity theory

AMS subject classification. 35J05, 35J25, 65N38, 78A45

1. introduction. Acoustic and electromagnetic scattering problems are often treated with boundary integral equation (BIE) methods. In a time-harmonic acoustic setting, the relevant boundary integral operators (BIOs) include the classical single layer and double layer potential operators and, more importantly, the combined field Helmholtz operators $A^{\prime}$ (see (1.4)) and $A$ (see (1.3)); the former is attributed to Burton \& Miller, [9], while the latter is commonly associated with the names of Brakhage \& Werner [3], Leis [15], and Panič [23]. These BIOs depend in a non-linear way on the wavenumber $k$ under consideration. Yet, especially in the high-frequency regime of large $k$, an understanding of how the boundary integral operators (BIOs) and the solutions of the BIEs depend on $k$ is important for various purposes, for example, for the design and analysis of efficient numerical schemes based on such BIEs. The present paper is devoted to a detailed analysis of the mapping properties of the BIOs $A$ and $A^{\prime}$ emphasizing the $k$-dependence.

The above mentioned reference have shown for smooth geometries (see [5] for the extension to Lipschitz geometries) that the combined field BIOs $A$ and $A^{\prime}$ are invertible on scales of Sobolev spaces. However, the $k$-dependence of these norms was left unspecified. For the special case of circular and spherical geometries the single and double layer potentials can be simultaneously diagonalized by Fourier techniques so that (using intricate large argument and large order asymptotics of Bessel functions) a complete $k$-explicit analysis of the operators $A, A^{\prime}$ and their inverses is possible, [11]. In this special cases, even $k$-uniform $L^{2}$-ellipticity is proved in [11]. More generally, [7] establishes for star-shaped domains $k$-uniform bounds for the norms $\left\|A^{-1}\right\|_{L^{2} \leftarrow L^{2}}$ and $\left\|\left(A^{\prime}\right)^{-1}\right\|_{L^{2} \leftarrow L^{2}}$. Estimates for $\|A\|_{L^{2} \leftarrow L^{2}}$ and $\left\|A^{\prime}\right\|_{L^{2} \leftarrow L^{2}}$, which depend on $k$, are provided in [4]. Norm bounds alone, however, are not sufficient for a sharp stability of analysis discretizations of the operators $A$ and $A^{\prime}$, especially in the context of high order methods. This observation was the starting point of the present paper, which

*INSTITUT FÜR ANALYSIS UND SCIENTIFIC COMPUTING, TECHNISCHE UNIVERSITÄT WIEN, AUSTRIA (MELENK@TUWIEN.AC.AT). 
provides a significantly more refined regularity theory for the operators $A, A^{\prime}$ and their inverses. In fact, the present analysis permits us to develop in the companion paper [17] a $k$-explicit convergence theory for the $h p$-version of the boundary element method ( $h p$-BEM).

Our $k$-explicit regularity theory takes the form of an additive decomposition of the operators into several terms with different mapping properties. Section 4 provides these decompositions for the classical single and double layer potentials. These operators are decomposed into three parts: the first part is the corresponding operator for the Laplace equation and therefore $k$-independent; the other two terms have smoothing properties but their operator norms depend on $k$ (we make this $k$-dependence explicit). Our principal decomposition results for the layer potential are for analytic geometries (see Theorems 4.3,4.4); however, it is also possible to obtain similar results for Lipschitz boundaries, which is worked out in Theorems 4.1, 4.2. Section 6 is at the heart of this paper and provides the additive decompositions for the inverses of the combined field operators in Theorems 6.11, 6.12; here, we restrict our attention to analytic geometries.

The result of the present paper have counterparts in the context of differential equations and finite elements. Indeed, analogous decomposition results have recently been obtained in $[21,22]$ for several Helmholtz boundary value problems.

The paper is organized as follows: the remainder of this first section introduces general notation and various boundary integral operators. Section 2 collects mapping properties of the classical single layer and double layer potential operators on Lipschitz domains. In particular, the limiting cases studied in Lemmata 2.1, 2.2 appear to be new. Section 3 studies the mapping properties of the Newton potential for the Helmholtz equation. Section 4 provides decomposition results for the Helmholtz single layer and double layer potential operators both for Lipschitz domains and domains with analytic boundaries. Section 5 applies the results of Section 4 to the combined field operators. The final Section 6 is a key section of the paper in that it provides decomposition results for the inverses of the combined field operators.

\section{1. notation and general assumptions.}

1.1.1. general notation. Let $\Omega \subset \mathbb{R}^{d}, d \in\{2,3\}$, be a bounded Lipschitz domain with a connected boundary. Throughout this work, we will futhermore assume for the case $d=2$ the scaling assumption $\operatorname{diam} \Omega<1$. We set $\Gamma:=\partial \Omega$ and $\Omega^{+}:=$ $\mathbb{R}^{d} \backslash \Omega$. Throughout the paper, we assume that the open ball $B_{R}:=B_{R}(0)$ of radius $R$ around the origin contains $\bar{\Omega}$, i.e., $\bar{\Omega} \subset B_{R}$. We set $\Omega_{R}:=\left(\Omega \cup \Omega^{+}\right) \cap B_{R}=B_{R} \backslash \Gamma$. We will denote by $\gamma_{0}^{\text {int }}$ and $\gamma_{0}^{\text {ext }}$ the interior and exterior trace operator on $\Gamma$. The interior and exterior co-normal derivative operators are denoted by $\gamma_{1}^{\text {int }}$, $\gamma_{1}^{\text {ext }}$, i.e., for sufficiently smooth functions $u$, we set $\gamma_{1}^{i n t} u:=\gamma_{0}^{i n t} \nabla u \cdot \vec{n}$ and $\gamma_{1}^{\text {ext }} u:=\gamma_{0}^{\text {ext }} \nabla u \cdot \vec{n}$, where, in both cases $\vec{n}$ is the unit normal vector point out of $\Omega$. As is standard, we introduce the jump operators

$$
[u]=\gamma_{0}^{e x t} u-\gamma_{0}^{i n t} u, \quad\left[\partial_{n} u\right]=\gamma_{1}^{e x t} u-\gamma_{1}^{i n t} u .
$$

For linear operators $\widetilde{\mathcal{A}}$ that map into spaces of piecewise defined functions, we define the operators $[\widetilde{\mathcal{A}}]$ and $\left[\partial_{n} \widetilde{\mathcal{A}}\right]$ in an analogous way, e.g., $[\widetilde{\mathcal{A}}] \varphi=[\widetilde{\mathcal{A}} \varphi]$. Sobolev spaces $H^{s}$ are defined in the standard way, $[1,27]$. We stress, however, that if an open set $\omega \subset \mathbb{R}^{d}$ consists of $m \in \mathbb{N}$ components of connectedness $\omega_{i}, i=1, \ldots, m$, then the space $H^{s}(\omega)$ can be identified with the product space $\prod_{i=1}^{m} H^{s}\left(\omega_{i}\right)$ equipped with the norm $\left(\sum_{i=1}^{m}\|u\|_{H^{s}\left(\omega_{i}\right)}^{2}\right)^{1 / 2}$. For a domain $\omega \subset \mathbb{R}^{d}$, we will also employ the 
Besov spaces $B_{1 / 2, \infty}^{s}(\omega)$, which are defined in the standard way by the real method of interpolation (see, e.g., $[2,27,28]$ ). Sets of analytic functions will play a very important role in our theory. We therefore introduce the following definition.

DEFINITION 1.1. For an open set $T$ and constant $C_{f}, \gamma_{f}>0$ we set

$$
\begin{aligned}
\mathfrak{A}\left(C_{f}, \gamma_{f}, T\right) & :=\left\{f \in L^{2}(T) \mid\left\|\nabla^{n} f\right\|_{L^{2}(T)} \leq C_{f} \gamma_{f}^{n} \max \{n+1,|k|\}^{n} \quad \forall n \in \mathbb{N}_{0}\right\} . \\
\text { Here, }\left|\nabla^{n} u(x)\right|^{2} & =\sum_{\alpha \in \mathbb{N}_{0}^{d}:|\alpha|=n} \frac{n !}{\alpha !}\left|D^{\alpha} u(x)\right|^{2} .
\end{aligned}
$$

For domains $\omega \subset \mathbb{R}^{d}$, it is convenient to introduce the $k$-dependent norm $\|u\|_{\mathcal{H}, \omega}$ by

$$
\|u\|_{\mathcal{H}, \omega}^{2}:=\|u\|_{L^{2}(\omega)}^{2}+k^{2}\|\nabla u\|_{L^{2}(\omega)}^{2} .
$$

Tubular neighborhoods $T$ of $\Gamma$ are open sets of such that $T \supset\left\{x \in \mathbb{R}^{d} \mid \operatorname{dist}(x, \Gamma)<\varepsilon\right\}$ for some $\varepsilon>0$.

Throughout the paper, we will use the following conventions:

Convention 1.2.

(i) We assume $|k| \geq k_{0}>0$ for some fixed $k_{0}>0$.

(ii) If the wavenumber $k$ appears outside the boundary integral operators and potentials such as $V_{k}$ and $\widetilde{V}_{k}$, then it is just a short-hand for $|k|$. In particular, $k$ stands for $|k|$ on the right-hand side of estimates. For example, $k \geq k_{0}$ stands for $|k| \geq k_{0}$.

1.1.2. layer potentials. In recent years, boundary element methods (BEM) and BIOs have been made accessible to a wider audience through several monographs, e.g., $[12,18,24,26]$. We refer to these books for more information about the operators studied here.

We denote by $V, K, K^{\prime}$ the usual single layer, double layer, and adjoint double layer operators for the Helmholtz equation. The single layer and double layer potentials are denoted by $\widetilde{V}$ and $\widetilde{K}$. More specifically, we define the Helmholtz kernel $G_{k}$ by

$$
\begin{aligned}
G_{k}(x, y) & :=\left\{\begin{array}{ll}
\frac{\mathbf{i}}{4} H_{0}^{(1)}(k|x-y|), & d=2, \\
\frac{e^{\mathbf{k} k|x-y|}}{4 \pi|x-y|}, & d=3,
\end{array} \text { for } k>0,\right. \\
G_{k} & :=\overline{G_{-k}} \quad \text { for } k<0,
\end{aligned}
$$

where $H_{0}^{(1)}$ is the first kind Hankel function of order zero. The limiting case $k=0$ corresponds to the Laplace operator and is defined as $G_{0}(x, y)=-1 /(2 \pi) \ln |x-y|$ for the case $d=2$ and $G_{0}(x, y)=1 /(4 \pi|x-y|)$ for the case $d=3$. The potential operators $\widetilde{V}$ and $\widetilde{K}$ are defined by

$(\widetilde{V} \varphi)(x):=\int_{\Gamma} G_{k}(x, y) \varphi(y) d s_{y}, \quad(\widetilde{K} \varphi)(x):=\int_{\Gamma} \partial_{n_{y}} G_{k}(x, y) \varphi(y) d s_{y}, \quad x \in \mathbb{R}^{d} \backslash \Gamma$.

From these potentials, the single layer, double layer, and adjoint double layer operators are defined as follows:

$$
V:=\gamma_{0}^{i n t} \widetilde{V}, \quad K:=\frac{1}{2}\left(\gamma_{0}^{i n t} \widetilde{K}+\gamma_{0}^{e x t} \widetilde{K}\right), \quad K^{\prime}:=\gamma_{1}^{i n t} \widetilde{V}-\frac{1}{2} \operatorname{Id} .
$$

If need be, we will write $V_{k}, K_{k}, K_{k}^{\prime}$ to clarify the $k$-dependence. We mention in passing that for $k \neq 0$, the potentials $\widetilde{V}_{k}$ and $\widetilde{K}_{k}$ are solutions of the homogeneous 
Helmholtz equation on $\mathbb{R}^{d} \backslash \Gamma$; for $k>0$ they satisfy the outgoing Sommerfeld radiation condition while for $k<0$, they satisfy the incoming radiation condition.

We finally turn to the definition of adjoint operators. We have for all $k \in \mathbb{R}$ for the $L^{2}(\Gamma)$ scalar product and all $\varphi, \psi \in H^{1 / 2}(\Gamma)$ :

$$
\begin{aligned}
\left(V_{k} \varphi, \psi\right)_{L^{2}(\Gamma)} & =\left(\varphi, V_{-k} \psi\right)_{L^{2}(\Gamma)}, \\
\left(K_{k} \varphi, \psi\right)_{L^{2}(\Gamma)} & =\left(\varphi, K_{-k}^{\prime} \psi\right)_{L^{2}(\Gamma)},
\end{aligned}
$$

i.e., the adjoints of $V_{k}$ and $K_{k}$ are $V_{-k}$ and $K_{-k}^{\prime}$, respectively. It is worth pointing out that we have the connections $\widetilde{V}_{-k} \bar{\varphi}=\overline{\widetilde{V}_{k} \varphi}$ and $\widetilde{K}_{-k} \bar{\varphi}=\overline{\widetilde{K}_{k} \varphi}$.

1.1.3. combined field operators. For a coupling parameter $\eta \in \mathbb{R} \backslash\{0\}$ we consider four combined field operators. The operator $A$ has one of the following two forms:

$$
\begin{aligned}
& A=A_{k}=-\frac{1}{2}+K-\mathbf{i} \eta V \\
& A=A_{k}=\frac{1}{2}+K-\mathbf{i} \eta V .
\end{aligned}
$$

The operator $A^{\prime}$ has one of the following two forms:

$$
\begin{aligned}
& A^{\prime}=A_{k}^{\prime}=-\frac{1}{2}+K^{\prime}+\mathbf{i} \eta V \\
& A^{\prime}=A_{k}^{\prime}=\frac{1}{2}+K^{\prime}+\mathbf{i} \eta V .
\end{aligned}
$$

We use the same notation for the operators in $(1.3 \mathrm{a}),(1.3 \mathrm{~b})$ and $(1.4 \mathrm{a}),(1.4 \mathrm{~b})$ since most of our results will be valid for both cases.

In order to avoid keeping track of the precise dependence of various constants on $\eta$, we assume throughout this paper that

$$
C_{\eta}^{-1}|k| \leq|\eta| \leq C_{\eta}|k|
$$

for some fixed $C_{\eta}>0$.

2. properties of the Laplace single and double layer potentials. In this section, we collect some mapping properties of the potential operators $\widetilde{V}_{0}$ and $\widetilde{K}_{0}$ for the Laplace equation.

2.1. Lipschitz domains. For Lipschitz domains $\Omega$ and $-1 \leq s \leq 1$ one can define the Sobolev spaces $H^{s}(\Gamma)$ intrinsically. It is then known (see also Lemmata 2.1, 2.2 below) that for $|s| \leq 1 / 2$ the operators

$$
\begin{aligned}
& \widetilde{V}_{0}: H^{-1 / 2+s}(\Gamma) \rightarrow H^{1}\left(B_{R}\right) \cap H^{1+s}\left(\Omega_{R}\right) \\
& \widetilde{K}_{0}: H^{1 / 2+s}(\Gamma) \rightarrow H^{1+s}\left(\Omega_{R}\right)
\end{aligned}
$$

are bounded linear operators (relevant literature includes [10,13,14,29]; see also Lemmata $2.1,2.2$ below). The following Lemma 2.1 clarifies into what space of functions defined on the ball $B_{R}$ (as opposed to $\Omega_{R}$ ) the potential operator $\widetilde{V}_{0}$ maps elements in the limiting cases $s= \pm 1 / 2$ :

LEMma 2.1 (mapping properties of $\widetilde{V}_{0}$ ). For $-1 / 2<s<1 / 2$ we have that $\widetilde{V}_{0}$ : $H^{-1 / 2+s}(\Gamma) \rightarrow H^{1+s}\left(B_{R}\right)$ is a bounded linear operator. The limiting cases $s= \pm 1 / 2$ take the forms $\widetilde{V}_{0}: H^{-1}(\Gamma) \rightarrow B_{2, \infty}^{1 / 2}\left(B_{R}\right)$ and $\widetilde{V}_{0}: L^{2}(\Gamma) \rightarrow B_{2, \infty}^{3 / 2}\left(B_{R}\right)$. 
Proof. The result for $-1 / 2<s<1 / 2$ are known in the literature (see, e.g., [18]). The proofs of the limiting cases $s= \pm 1 / 2$ are relegated to Appendix A.

The potential operator $\widetilde{K}_{0}$ produces functions that jump across $\Gamma$. This implies that, viewed as a function on the ball $B_{R}$, one cannot hope for more regularity than $\widetilde{K}_{0} \varphi \in$ $B_{2, \infty}^{1 / 2}\left(B_{R}\right)$; this is indeed the case for the limiting case $s=-1 / 2$ :

LEMMA 2.2 (mapping properties of $\widetilde{K}_{0}$ ). For $-1 / 2 \leq s \leq 1 / 2$ we have $\widetilde{K}_{0}$ : $H^{1 / 2+s}(\Gamma) \rightarrow H^{1+s}\left(\Omega_{R}\right)$. For the limiting case $s=-1 / 2$ we have the additional result $\widetilde{K}_{0}: L^{2}(\Gamma) \rightarrow B_{2, \infty}^{1 / 2}\left(B_{R}\right)$.

Proof. See Appendix A.

2.2. smooth domains. The mapping properties given in (2.1) are restricted to the range $|s| \leq 1 / 2$ for Lipschitz domains. For smooth domains, the range can be extended, for example, to include all $s \geq-1$. To that end, we note

Lemma 2.3. Let $\Gamma$ be of class $C^{\infty}$. Then there exists $C>0$ depending only on $\Omega$ and $R$ such that for $\varphi \in H^{1 / 2}(\Gamma)$ there holds

$$
\left\|\widetilde{V}_{0} \varphi\right\|_{L^{2}\left(\Omega_{R}\right)} \leq C\|\varphi\|_{H^{-3 / 2}(\Gamma)}, \quad\left\|\widetilde{K}_{0} \varphi\right\|_{L^{2}\left(\Omega_{R}\right)} \leq C\|\varphi\|_{H^{-1 / 2}(\Gamma)} .
$$

Proof. Set $u:=\widetilde{V}_{0} \varphi$. We only aim at estimating $\|u\|_{L^{2}(\Omega)}$ since $\|u\|_{L^{2}\left(\Omega_{R} \backslash \Omega\right)}$ is estimated similarly. To that end, let $w \in H^{2}(\Omega)$ solve

$$
-\Delta w=u \quad \text { in } \Omega, \quad \partial_{n} w=0 \quad \text { on } \Gamma .
$$

Then $w \in H^{2}(\Omega)$ together with $\|w\|_{H^{2}(\Omega)} \lesssim\|u\|_{L^{2}(\Omega)}$ and therefore

$$
\|u\|_{L^{2}(\Omega)}^{2}=\left|\int_{\Gamma} \gamma_{1}^{i n t} u w\right| \lesssim\left\|\gamma_{1}^{i n t} u\right\|_{H^{-3 / 2}(\Gamma)}\|w\|_{H^{2}(\Omega)} \lesssim\left\|\gamma_{1}^{i n t} u\right\|_{H^{-3 / 2}(\Gamma)}\|u\|_{L^{2}(\Omega)} .
$$

Next, we use the representation $\gamma_{1}^{\text {int } u} u=\gamma_{1}^{\text {int }} \widetilde{V}_{0} \varphi=\left(\frac{1}{2}+K_{0}^{\prime}\right) \varphi$ and [18, Thm. 7.2] to bound $\left\|\gamma_{1}^{i n t} u\right\|_{H^{-3 / 2}(\Gamma)} \leq C\|\varphi\|_{H^{-3 / 2}(\Gamma)}$.

We proceed in a similar manner to bound $\left\|\widetilde{K}_{0} \varphi\right\|_{L^{2}(\Omega)}$. Let $u=\left.\left(\widetilde{K}_{0} \varphi\right)\right|_{\Omega}$ and let $w \in H^{2}(\Omega) \cap H_{0}^{1}(\Omega)$ solve

$$
-\Delta w=u \quad \text { in } \Omega,\left.\quad w\right|_{\Gamma}=0 .
$$

Then $\|w\|_{H^{2}(\Omega)} \lesssim\|u\|_{L^{2}(\Omega)}$ and therefore

$$
\|u\|_{L^{2}(\Omega)}^{2}=\left|\int_{\Gamma} \gamma_{1}^{i n t} w \gamma_{0} u\right| \lesssim\|u\|_{H^{-1 / 2}(\Gamma)}\left\|\gamma_{1}^{i n t} w\right\|_{H^{1 / 2}(\Gamma)} \lesssim\|u\|_{H^{-1 / 2}(\Gamma)}\|u\|_{L^{2}(\Gamma)} .
$$

From the representation $\gamma_{0}^{\text {int }} u=\left(-\frac{1}{2}+K_{0}\right) \varphi$ and the mapping properties of $K_{0}$ on smooth domains, $\left[18\right.$, Thm. 7.2], we get again $\|u\|_{H^{-1 / 2}(\Gamma)} \leq C\|\varphi\|_{H^{-1 / 2}(\Gamma)}$. Lemma 2.3 allows us to extend the operators $\widetilde{V}_{0}$ and $\widetilde{K}_{0}$ to operators defined on $H^{-3 / 2}(\Gamma)$ and $H^{-1 / 2}(\Gamma)$ respectively. We thus have

LemMA 2.4. Let $\Gamma$ be of class $C^{\infty}$. Then the operators $\widetilde{V}_{0}$ and $\widetilde{K}_{0}$ are bounded linear operators

$$
\widetilde{V}_{0}: H^{-1 / 2+s}(\Gamma) \rightarrow H^{1+s}\left(\Omega_{R}\right), \quad \widetilde{K}_{0}: H^{1 / 2+s}(\Gamma) \rightarrow H^{1+s}\left(\Omega_{R}\right)
$$

for every $s \geq-1$ and every $R>0$ such that $\bar{\Omega} \subset B_{R}$.

Proof. The case $s>-1 / 2$ is shown in [18, Cor. 6.14]. The case $s=-1$ follows from Lemma 2.3. An interpolation argument then provided the intermediate range $-1 \leq s \leq-1 / 2$. 
2.3. invertibility properties. For future reference, we recall the following results:

Lemma 2.5. Let $\Gamma$ be smooth and $\alpha \in \mathbb{R} \backslash\{0\}$ be fixed. If $d=2$, assume additionally that $\operatorname{diam} \Omega<1$. Then:

(i) $-\frac{1}{2}+K_{0}: H^{s}(\Gamma) \rightarrow H^{s}(\Gamma)$ is boundedly invertible for $s \geq 0$.

(ii) $\frac{1}{2}+K_{0}+\mathbf{i} \alpha V_{0}: H^{s}(\Gamma) \rightarrow H^{s}(\Gamma)$ is boundedly invertible for $s \geq 0$.

(iii) $-\frac{1}{2}+K_{0}^{\prime}: H^{s}(\Gamma) \rightarrow H^{s}(\Gamma)$ is boundedly invertible for $s \geq-1 / 2$.

(iv) $\frac{1}{2}+K_{0}^{\prime}+\mathbf{i} \alpha V_{0}: H^{s}(\Gamma) \rightarrow H^{s}(\Gamma)$ is boundedly invertible for $s \geq-1 / 2$.

Proof. See, e.g., [16, Appendix D].

3. Properties of the Helmholtz Newton potential. A key ingredient of our decomposition of the operators $\widetilde{V}, \widetilde{K}$, and $A, A^{\prime}$ are low pass and high pass filters that we introduce now:

LEMma 3.1 (full space frequency splitting). Let $q \in(0,1)$. Then one can construct linear operators $H_{\mathbb{R}^{d}}$ and $L_{\mathbb{R}^{d}}$ defined on $L^{2}\left(\mathbb{R}^{d}\right)$ with the following properties:

(i) $H_{\mathbb{R}^{d}}+L_{\mathbb{R}^{d}}=\mathrm{Id}$

(ii) $\left\|H_{\mathbb{R}^{d}} f\right\|_{H^{s^{\prime}\left(\mathbb{R}^{d}\right)}} \leq C_{s, s^{\prime}}\left(q k^{-1}\right)^{s-s^{\prime}}\|f\|_{H^{s}\left(\mathbb{R}^{d}\right)}$ for all $0 \leq s^{\prime} \leq s$ and $f \in H^{s}\left(\mathbb{R}^{d}\right)$

(iii) $L_{\mathbb{R}^{d}} f$ is entire and

$$
\left\|\nabla^{n} L_{\mathbb{R}^{d}} f\right\|_{L^{2}\left(\mathbb{R}^{d}\right)} \leq C(\gamma k)^{n}\|f\|_{L^{2}\left(\mathbb{R}^{d}\right)} \quad \forall n \in \mathbb{N}_{0}
$$

Here, the constants $C, \gamma$ depend on the choice of $q$ and $s$ but are independent of $k \geq k_{0}$.

Proof. See [21, Lemmata 4.2, 4.3] for details. A sketch of the construction is as follows: The operators $H_{\mathbb{R}^{d}}$ and $L_{\mathbb{R}^{d}}$ are defined in terms the Fourier transformation $\mathcal{F}$ : $L^{2}\left(\mathbb{R}^{2}\right) \rightarrow L^{2}\left(\mathbb{R}^{d}\right)$ by $\mathcal{F}\left(H_{\mathbb{R}^{d}}(f)\right):=\chi_{\mathbb{R}^{d} \backslash B_{k \eta}(0)} \mathcal{F}(f)$ and $\mathcal{F}\left(L_{\mathbb{R}^{d}}(f)\right):=\chi_{B_{k \eta}(0)} \mathcal{F}(f)$. Here, $\eta>1$ is a parameter that is selected depending on the chosen $q \in(0,1)$ and $\chi_{E}$ denotes the characteristic function of the set $E \subset \mathbb{R}^{d}$.

The Newton potential $N_{k}(f)$ of $f \in L^{2}\left(\mathbb{R}^{d}\right)$ with compact support is defined by

$$
N_{k}(f):=G_{k} \star f .
$$

It is the solution of the inhomogeneous Helmholtz equation with right-hand side $f$ and satisfies the outgoing radiation condition if $k>0$ and the incoming radiation condition if $k<0$. For $N_{k}$ we have the following decomposition result:

LEMMA 3.2 (mapping properties of $N_{k}$ ). For every $f \in L^{2}\left(\mathbb{R}^{d}\right)$ there holds

$$
\left\|N_{k}(f)\right\|_{\mathcal{H}, B_{R}}+k^{-1}\left\|N_{k}(f)\right\|_{H^{2}\left(B_{R}\right)} \leq C_{R}\|f\|_{L^{2}\left(\mathbb{R}^{d}\right)}
$$

Additionally, the following decomposition result holds: Let $q \in(0,1)$ be arbitrary. Then the high frequency operator $H_{\mathbb{R}^{d}}$ and the low frequency operator $L_{\mathbb{R}^{d}}$ can be chosen such that for $s \geq 0$ and $0 \leq s^{\prime} \leq s+2$ the function $N_{k}\left(H_{\mathbb{R}^{d}} f\right)$ satisfies

$$
\left\|N_{k}\left(H_{\mathbb{R}^{d}} f\right)\right\|_{H^{s^{\prime}}\left(B_{R}\right)} \leq C_{s, s^{\prime}}\left(q k^{-1}\right)^{2+s-s^{\prime}}\|f\|_{H^{s}\left(\mathbb{R}^{d}\right)} .
$$

The constant $C_{s, s^{\prime}}$ is independent of $q \in(0,1)$ and $k \geq k_{0}$. The function $N_{k}\left(L_{\mathbb{R}^{d}} f\right)$ is entire and satisfies

$$
\left\|\nabla^{n} N_{k}\left(L_{\mathbb{R}^{d}} f\right)\right\|_{L^{2}\left(B_{R}\right)} \leq C(\gamma k)^{n-1}\|f\|_{L^{2}\left(\mathbb{R}^{d}\right)} \quad \forall n \in \mathbb{N}_{0} .
$$

Here, the constants $C, \gamma$ are independent of $k \geq k_{0}$ but depend on $q$. 
Proof. The estimate (3.2) is shown in [22, Lemma 3.5]. Inspection of the procedure in [22, Lemma 3.5] reveals that the function $v_{\mathcal{A}}$ in [22, Lemma 3.5] coincides with $N_{k}\left(L_{\mathbb{R}^{d}} f\right)$, which shows (3.4). Finally, [22, Lemma 3.5] shows (3.3) for the case $s=0$. Inspection of the proof shows that it can be extended in a straight forwards way to the case $s>0$.

An interpolation argument allows us to infer the following result:

COROllary 3.3. Let $s \geq 0$ and $s \notin \mathbb{N}_{0}$. Fix a cut-off function $\chi$ with $\operatorname{supp} \chi \subset B_{2 R}$. Then for all $f \in B_{2, \infty}^{s}\left(B_{2 R}\right)$

$$
\begin{aligned}
\left\|N_{k}\left(H_{\mathbb{R}^{d}}(\chi f)\right)\right\|_{H^{s^{\prime}}\left(B_{R}\right)} & \leq C_{s, s^{\prime}}\left(q k^{-1}\right)^{2+s-s^{\prime}}\|f\|_{B_{2, \infty}^{s}\left(B_{2 R}\right)}, \quad 0 \leq s^{\prime}<2+s \\
\left\|N_{k}\left(H_{\mathbb{R}^{d}}(\chi f)\right)\right\|_{B_{2, \infty}^{2+s}\left(B_{R}\right)} & \leq C_{s}\|f\|_{B_{2, \infty}^{s}\left(B_{2 R}\right)} .
\end{aligned}
$$

Proof. The operator $f \mapsto N_{k}\left(H_{\mathbb{R}^{d}} \chi f\right)$ is linear and, for every $t \geq 0$, we have by Lemma 3.2

$$
\begin{aligned}
\left\|N_{k}\left(H_{\mathbb{R}^{d}} \chi f\right)\right\|_{H^{t+2}\left(B_{R}\right)} & \leq C_{t}\|f\|_{H^{t}\left(B_{2 R}\right)}, \\
\left\|N_{k}\left(H_{\mathbb{R}^{d}} \chi f\right)\right\|_{L^{2}\left(B_{R}\right)} & \leq C_{t}\left(q k^{-1}\right)^{2+t}\|f\|_{H^{t}\left(B_{2 R}\right)},
\end{aligned}
$$

for a constant $C_{t}>0$ that depends solely on $t, R$, and $\chi$. Since the spaces $B_{2, \infty}^{s}$ are defined as interpolation spaces between standard Sobolev spaces, the estimates (3.7) imply (3.6). Since $\left(L^{2}\left(B_{R}\right), L^{2}\left(B_{R}\right)\right)_{\theta, \infty}=L^{2}\left(B_{R}\right)$ for every $\theta \in(0,1)$, the estimate (3.5) for the special case $s^{\prime}=0$ follows also from an interpolation argument and (3.8). Finally, the general case in (3.5) follows from the interpolation inequality $\|z\|_{H^{\theta(s+2)}} \leq C\|z\|_{L^{2}}^{1-\theta}\|z\|_{B_{2, \infty}^{s+2}}^{\theta}$ for $s+2>0$ and $\theta \in(0,1)$.

4. decomposition of layer potentials. The present section focuses on the mapping properties of the layer potentials $\widetilde{V}$ and $\widetilde{K}$ with particular emphasis on making the $k$-dependence explicit. We do this through an additive decomposition of $\widetilde{V}$ and $\widetilde{K}$ into a leading order part that corresponds to the Laplace operator (i.e., $\widetilde{V}_{0}$ and $\widetilde{K}_{0}$ ) and regularizing parts.

We present two different types of decompositions: the first type is done for Lipschitz domains and formulated in Subsection 4.1. Since the regularizing parts are defined as solutions of transmission problems, the limited regularity of Lipschitz domains imposes restrictions on the Sobolev range for which the decomposition can be done in a meaningful way. We therefore consider in Section 4.2 the case of domains with analytic boundary, where, by a modification of the procedure of Section 4.1, decompositions are obtained that are valid for large ranges of Sobolev spaces.

\section{1. decomposition of layer potentials: Lipschitz domains.}

4.1.1. decomposition of the single layer potential. THEOREM 4.1 (decomposition of $\widetilde{V}$, Lipschitz domain). Let $q \in(0,1)$ be given. Then one can write

$$
\widetilde{V}=\widetilde{V}_{0}+\widetilde{S}_{V}+\widetilde{\mathcal{A}}_{V}
$$

where for every $-1 / 2<s<1 / 2$ the linear operators $\widetilde{S}_{V}: H^{-1 / 2+s}(\Gamma) \rightarrow H^{3+s}\left(B_{R}\right)$ and $\widetilde{\mathcal{A}}_{V}: H^{-1 / 2+s}(\Gamma) \rightarrow H^{3+s}\left(B_{R}\right)$ satisfy the following bounds:

$$
\begin{aligned}
& \left\|\widetilde{S}_{V} \varphi\right\|_{H^{s^{\prime}\left(B_{R}\right)}} \leq C_{s, s^{\prime}} q^{2}\left(q k^{-1}\right)^{1+s-s^{\prime}}\|\varphi\|_{H^{-1 / 2+s}(\Gamma)}, \quad 0 \leq s^{\prime} \leq 3+s, \\
& \left\|\nabla^{n} \widetilde{\mathcal{A}}_{V} \varphi\right\|_{L^{2}\left(B_{R}\right)} \leq C(\gamma k)^{n+1}\left\|\widetilde{V}_{0} \varphi\right\|_{L^{2}\left(B_{R}\right)} \leq C(\gamma k)^{n+1}\|\varphi\|_{H^{-1}(\Gamma)} \quad \forall n \in \mathbb{N}_{0} \text {. }
\end{aligned}
$$


Here, the constant $C_{s, s^{\prime}}$ is independent of $q$ and $k \geq k_{0}$. The constants $C, \gamma$ are independent of $k \geq k_{0}$ but depend on $q$.

For $s= \pm 1 / 2$ we have that $\widetilde{S}_{V}: H^{-1 / 2+s}(\Gamma) \rightarrow B_{2, \infty}^{3+s}\left(B_{R}\right)$ and $\widetilde{\mathcal{A}}_{V}: H^{-1 / 2+s}(\Gamma) \rightarrow$ $B_{2, \infty}^{3+s}\left(B_{R}\right)$ satisfy the following bounds:

$$
\begin{aligned}
\left\|\widetilde{S}_{V} \varphi\right\|_{H^{s^{\prime}}\left(B_{R}\right)} & \leq C_{s, s^{\prime}} q^{2}\left(q k^{-1}\right)^{1+s-s^{\prime}}\|\varphi\|_{H^{-1 / 2+s}(\Gamma)}, \quad 0 \leq s^{\prime}<3+s, \\
\left\|\widetilde{S}_{V} \varphi\right\|_{B_{2, \infty}^{s+3}\left(B_{R}\right)} & \leq C_{s} q^{2}\left(q k^{-1}\right)^{-2}\|\varphi\|_{H^{-1 / 2+s}(\Gamma)}, \\
\left\|\nabla^{n} \widetilde{\mathcal{A}}_{V} \varphi\right\|_{L^{2}\left(B_{R}\right)} & \leq C(\gamma k)^{n+1}\left\|\widetilde{V}_{0} \varphi\right\|_{L^{2}\left(B_{R}\right)} \leq C(\gamma k)^{n+1}\|\varphi\|_{H^{-1}(\Gamma)} \quad \forall n \in \mathbb{N}_{0} .
\end{aligned}
$$

Proof. We will exploit density of $H^{1 / 2}(\Gamma)$ in $H^{-1 / 2+s}(\Gamma)$ for $-1 / 2 \leq s \leq 1 / 2$. Let therefore $\varphi \in H^{1 / 2}(\Gamma)$ be given. Set $u:=\widetilde{V} \varphi$ and $u_{0}:=\widetilde{V}_{0} \varphi$. Let $\chi$ be a smooth cut-off function with supp $\chi \subset B_{2 R}$ and $\left.\chi\right|_{B_{R}} \equiv 1$. Then the function $\tilde{u}:=u-\chi u_{0}$ satisfies

$$
\begin{array}{rlrl}
-\Delta \tilde{u}-k^{2} \tilde{u} & =f:=-(\Delta \chi) u_{0}-2 \nabla \chi \cdot \nabla u_{0}+k^{2} \chi u_{0} \quad \text { in } \Omega \cup \Omega^{+}, \\
{[\tilde{u}]} & =0 & & \text { on } \Gamma\left(\text { in } H^{1 / 2}(\Gamma)\right), \\
{\left[\partial_{n} \tilde{u}\right]} & =0 & & \text { on } \Gamma\left(\text { in } H^{-1 / 2}(\Gamma)\right), \\
\tilde{u} & & \text { satisfies a radiation condition at } \infty,
\end{array}
$$

and $f$ has compact support. The mapping properties of $\widetilde{V}_{0}$ on Lipschitz domains of Lemma 2.1 imply for $-1 / 2 \leq s \leq 1 / 2$ :

$$
\begin{array}{rlrl}
\left\|u_{0}\right\|_{H^{1+s}\left(B_{R}\right)} & \leq C\|\varphi\|_{H^{-1 / 2+s}(\Gamma)}, & & -1 / 2<s<1 / 2, \\
\left\|u_{0}\right\|_{B_{2, \infty}^{1+s}\left(B_{R}\right)} \leq C\|\varphi\|_{H^{-1 / 2+s}(\Gamma)}, & & s= \pm 1 / 2 .
\end{array}
$$

We have therefore an explicit solution formula for $\tilde{u}$, namely,

$$
\tilde{u}=N_{k}(f)
$$

Hence, we have the representation

$$
u=\chi u_{0}+N_{k}(f)=\chi u_{0}+N_{k}\left(H_{\mathbb{R}^{d}} f\right)+N_{k}\left(L_{\mathbb{R}^{d}} f\right)=: \chi u_{0}+\widetilde{S}_{V} \varphi+\widetilde{\mathcal{A}}_{V} \varphi,
$$

where the parameter $q$ in the definition of $H_{\mathbb{R}^{d}}$ is still at our disposal.

We first consider the regularity of $\widetilde{S}_{V}$. In view of Lemma 3.2 and Corollary 3.3 we have to analyze the regularity properties of $f$. By interior regularity, we have that $u_{0}$ is analytic away from $\Gamma$, and we get for $s= \pm 1 / 2$ :

$$
\|f\|_{B_{2, \infty}^{1+s}\left(B_{2 R}\right)} \leq C k^{2}\|\varphi\|_{H^{-1 / 2+s}(\Gamma)} .
$$

Next, the support properties of $f$ imply that $f=\chi^{\prime} f$ for some smooth cut-off function $\chi^{\prime}$. Hence, Corollary 3.3 implies for $s= \pm 1 / 2$

$$
\begin{aligned}
\left\|N_{k}\left(H_{\mathbb{R}^{d}} f\right)\right\|_{L^{2}\left(B_{R}\right)} & \leq C\left(q k^{-1}\right)^{3+s} k^{2}\|\varphi\|_{H^{-1 / 2+s}(\Gamma)}, \\
\left\|N_{k}\left(H_{\mathbb{R}^{d}} f\right)\right\|_{B_{2, \infty}^{3+s}\left(B_{R}\right)} & \leq C k^{2}\|\varphi\|_{H^{-1 / 2+s}(\Gamma)} .
\end{aligned}
$$

Interpolation then allows us to conclude for $-1 / 2<s<1 / 2$

$$
\begin{aligned}
\left\|N_{k}\left(H_{\mathbb{R}^{d}} f\right)\right\|_{L^{2}\left(B_{R}\right)} & \leq C\left(q k^{-1}\right)^{3+s} k^{2}\|\varphi\|_{H^{-1 / 2+s}(\Gamma)}, \\
\left\|N_{k}\left(H_{\mathbb{R}^{d}} f\right)\right\|_{H^{3+s}\left(B_{R}\right)} & \leq C k^{2}\|\varphi\|_{H^{-1 / 2+s}(\Gamma)} .
\end{aligned}
$$


We have thus shown all the estimates for $\widetilde{\mathcal{S}}_{V}$ for the cases $s^{\prime}=0$ and $s^{\prime}=3+s$. For the remaining intermediate estimates, we simply use another interpolation argument. Specifically, for the case $-1 / 2<s<1 / 2$ we use the multiplicative interpolation inequality with $\theta=s^{\prime} /(3+s)$ to get

$$
\begin{aligned}
\left\|N_{k}\left(H_{\mathbb{R}^{d}} f\right)\right\|_{H^{s^{\prime}}\left(B_{R}\right)} & \leq C\left\|N_{k}\left(H_{\mathbb{R}^{d}} f\right)\right\|_{L^{2}\left(B_{R}\right)}^{1-\theta}\left\|N_{k}\left(H_{\mathbb{R}^{d}} f\right)\right\|_{H^{3+s}\left(B_{R}\right)}^{\theta} \\
& \leq C k^{2}\left(q k^{-1}\right)^{3+s-s^{\prime}}\|\varphi\|_{H^{-1 / 2+s}(\Gamma)} .
\end{aligned}
$$

Let us now turn to the $N_{k}\left(L_{\mathbb{R}^{d}} f\right)$. From Lemma 3.2 we get

$$
\begin{aligned}
\left\|\nabla^{n} N_{k}\left(L_{\mathbb{R}^{d}} f\right)\right\|_{L^{2}\left(B_{R}\right)} & \leq C(\gamma k)^{n-1}\|f\|_{L^{2}\left(B_{2 R}\right)} \leq C(\gamma k)^{n-1} k^{2}\left\|u_{0}\right\|_{L^{2}\left(B_{2 R}\right)} \\
& \leq C(\gamma k)^{n+1}\left\|\widetilde{V}_{0} \varphi\right\|_{L^{2}\left(B_{2 R}\right)} .
\end{aligned}
$$

Density of $H^{1 / 2}(\Gamma)$ in $H^{-1 / 2+s}(\Gamma)$ concludes the argument.

4.1.2. decomposition of the double layer potential. The method of proof of Theorem 4.1 is applicable to the double layer potential as well for the end point case $s=-1 / 2$ :

TheOREM 4.2 (decomposition of $\widetilde{K}$, Lipschitz domain). Let $\Omega \subset B_{R}$ be a Lipschitz domain and let $q \in(0,1)$ be given. Then

$$
\widetilde{K}=\widetilde{K}_{0}+\widetilde{S}_{K}+\widetilde{\mathcal{A}}_{K}
$$

where $\widetilde{S}_{K}: L^{2}(\Gamma) \rightarrow B_{2, \infty}^{5 / 2}\left(B_{R}\right)$ satisfies

$$
\begin{aligned}
\left\|\widetilde{S}_{K} \varphi\right\|_{B_{2, \infty}^{5 / 2}\left(B_{R}\right)} & \leq C k^{2}\|\varphi\|_{L^{2}(\Gamma)}, \\
\left\|\widetilde{S}_{K} \varphi\right\|_{L^{2}\left(B_{R}\right)} & \leq C q^{2}\left(q k^{-1}\right)^{1 / 2}\|\varphi\|_{L^{2}(\Gamma)} .
\end{aligned}
$$

Here, the constant $C$ is independent of $q$ and $k \geq k_{0}$. The linear operator $\widetilde{\mathcal{A}}_{K}$ : $L^{2}(\Gamma) \rightarrow B_{2, \infty}^{5 / 2}\left(B_{R}\right)$ maps into a space of analytic functions, viz.,

$$
\left\|\nabla^{n} \widetilde{\mathcal{A}}_{K} \varphi\right\|_{L^{2}\left(B_{R}\right)} \leq C(\gamma k)^{n+1}\left\|\widetilde{K}_{0} \varphi\right\|_{L^{2}\left(B_{R}\right)} \leq C(\gamma k)^{n+1}\|\varphi\|_{L^{2}(\Gamma)} \quad \forall n \in \mathbb{N}_{0} .
$$

Here, the constants $C, \gamma>0$ are independent of $k \geq k_{0}$ but may depend on $q$.

Proof. We proceed as in the proof of Theorem 4.1. This implies the form

$$
\widetilde{K}=\widetilde{K}_{0}+\widetilde{S}_{K}+\widetilde{\mathcal{A}}_{K}
$$

here, $\widetilde{S}_{K}$ and $\widetilde{\mathcal{A}}_{K}$ are defined by

$$
\widetilde{S}_{K} \varphi+\widetilde{\mathcal{A}}_{K} \varphi:=N_{k}\left(H_{\mathbb{R}^{d}} f\right)+N_{k}\left(L_{\mathbb{R}^{d}} f\right),
$$

where, for $u_{0}=\widetilde{K}_{0} \varphi$, the function $f$ is given by

$$
f=-\Delta \chi u_{0}+2 \nabla \chi \cdot \nabla u_{0}+k^{2} \chi u_{0}
$$

The mapping properties of $\widetilde{K}_{0}$ detailed in Lemma 2.2 imply $\widetilde{K}_{0} \varphi \in B_{2, \infty}^{1 / 2}\left(B_{2 R}\right)$. Proceeding as in the proof of Theorem 4.1 we arrive at

$$
\begin{aligned}
\left\|N_{k}\left(H_{\mathbb{R}^{d}} f\right)\right\|_{L^{2}\left(B_{R}\right)} & \leq C\left(q k^{-1}\right)^{2+1 / 2}\|f\|_{B_{2, \infty}^{1 / 2}\left(B_{2 R}\right)} \\
& \leq C q^{2} k^{-2}\left(q k^{-1}\right)^{1 / 2} k^{2}\left\|\widetilde{K}_{0} \varphi\right\|_{B_{2, \infty}^{1 / 2}\left(B_{2 R}\right)} \leq C q^{2}\left(q k^{-1}\right)^{1 / 2}\|\varphi\|_{L^{2}(\Gamma)}, \\
\left\|N_{k}\left(H_{\mathbb{R}^{d}} f\right)\right\|_{B_{2, \infty}^{5 / 2}\left(B_{R}\right)} & \leq C\|f\|_{B_{2, \infty}^{1 / 2}\left(B_{2 R}\right)} \leq C k^{2}\|\varphi\|_{L^{2}(\Gamma) .}
\end{aligned}
$$

The estimates for $\widetilde{A}_{K} \varphi$ are obtained in exactly the same way as in Theorem 4.1. 
4.2. decomposition of layer potentials: analytic boundaries. The method of proof in Theorems 4.1 and 4.2 relies on (Sobolev) regularity of $\widetilde{V}_{0} \varphi$ or $\widetilde{K}_{0} \varphi$ as a function on the ball $B_{2 R}$. However, these functions are only piecewise smooth (higher order derivatives jump across $\Gamma$ ), and the approach of Theorems 4.1, 4.2 could not exploit this piecewise smoothness. In order to exploit it, we need to modify the definition of the operators $\widetilde{S}_{V}$ and $\widetilde{S}_{K}$. Our approach to the construction of decompositions will rely on a regularity theory for transmission problem, where the transmission conditions are imposed on $\Gamma$. This requires regularity of $\Gamma$. We illustrate what kind of result may be expected for the case of analytic $\Gamma$.

THEOREM 4.3 (decomposition of $\widetilde{V}$, analytic boundary). Let $\Gamma$ be analytic and $q \in$ $(0,1)$. Then

$$
\widetilde{V}=\widetilde{V}_{0}+\widetilde{S}_{V, p w}+\widetilde{\mathcal{A}}_{V, p w}
$$

where the linear operators $\widetilde{S}_{V, p w}$ and $\widetilde{\mathcal{A}}_{V, p w}$ satisfy the following for every $s \geq-1$ :

(i) $\widetilde{S}_{V, p w}: H^{-1 / 2+s}(\Gamma) \rightarrow H^{2}\left(B_{R}\right) \cap H^{3+s}\left(\Omega_{R}\right)$ with

$$
\left\|\widetilde{S}_{V, p w} \varphi\right\|_{H^{s^{\prime}}\left(\Omega_{R}\right)} \leq C_{s^{\prime}, s} q^{2}\left(q k^{-1}\right)^{1+s-s^{\prime}}\|\varphi\|_{H^{-1 / 2+s}(\Gamma)}, \quad 0 \leq s^{\prime} \leq s+3 .
$$

Here, the constant $C_{s^{\prime}, s}>0$ is independent of $q$ and $k \geq k_{0}$.

(ii) $\widetilde{\mathcal{A}}_{V, p w}: H^{-1 / 2+s}(\Gamma) \rightarrow H^{2}\left(B_{R}\right)$ maps into a space of piecewise analytic functions and

$$
\left\|\nabla^{n} \widetilde{\mathcal{A}}_{V, p w} \varphi\right\|_{L^{2}\left(\Omega_{R}\right)} \leq C k \gamma^{n} \max \{n+1, k\}^{n}\|\varphi\|_{H^{-3 / 2}(\Gamma)} \quad \forall n \in \mathbb{N}_{0} .
$$

Here, the constants $C, \gamma>0$ are independent of $k \geq k_{0}$ but may depend on $q$. Proof. We start again as in the proof of Theorem 4.1. We have

$$
f=-(\Delta \chi) u_{0}-2 \nabla \chi \cdot \nabla u_{0}+k^{2} \chi u_{0}
$$

where $u_{0}=\widetilde{V}_{0} \varphi$ and $\chi$ is the cut-off function of Theorem 4.1. By the mapping properties of $\widetilde{V}_{0}$ (cf. Lemma 2.4), we have that $f$ is piecewise in $H^{1+s}$. More specifically,

$$
\|f\|_{H^{1+s}\left(\Omega_{2 R}\right)} \leq C k^{2}\|\varphi\|_{H^{-1 / 2+s}(\Gamma)} .
$$

Let $E_{\Omega}$ and $E_{\Omega^{+}}$be the Stein extension operators (see [25, Chap. VI.3, Thm. 5]) for the sets $\Omega$ and $\Omega^{+}$. Additionally, let $\chi_{\Omega}$ and $\chi_{\Omega^{+}}$be the characteristic functions of $\Omega$ and $\Omega^{+}$. We observe

$$
\begin{aligned}
& f=H_{\mathbb{R}^{d}}\left(E_{\Omega}\left(f \chi_{\Omega}\right)\right)+L_{\mathbb{R}^{d}}\left(E_{\Omega}\left(f \chi_{\Omega}\right)\right) \quad \text { in } \Omega, \\
& f=H_{\mathbb{R}^{d}}\left(E_{\Omega^{+}}\left(f \chi_{\Omega^{+}}\right)\right)+L_{\mathbb{R}^{d}}\left(E_{\Omega^{+}}\left(f \chi_{\Omega^{+}}\right)\right) \quad \text { in } \Omega^{+} .
\end{aligned}
$$

These formulas suggest to write $f$ in the form $f=f_{H^{1+s}}+f_{\mathcal{A}, p w}$, where

$$
\begin{array}{llrl}
\left.f_{H^{1+s}}\right|_{\Omega}=\left.H_{\mathbb{R}^{d}}\left(E_{\Omega}\left(f \chi_{\Omega}\right)\right)\right|_{\Omega}, & & \left.f_{H^{1+s}}\right|_{\Omega^{+}}=\left.H_{\mathbb{R}^{d}}\left(E_{\Omega^{+}}\left(f \chi_{\Omega^{+}}\right)\right)\right|_{\Omega^{+}}, \\
\left.f_{\mathcal{A}, p w}\right|_{\Omega}=\left.L_{\mathbb{R}^{d}}\left(E_{\Omega}\left(f \chi_{\Omega}\right)\right)\right|_{\Omega}, & & \left.f_{\mathcal{A}, p w}\right|_{\Omega^{+}}=\left.L_{\mathbb{R}^{d}}\left(E_{\Omega^{+}}\left(f \chi_{\Omega^{+}}\right)\right)\right|_{\Omega^{+}},
\end{array}
$$

The properties of $H_{\mathbb{R}^{d}}$ and $L_{\mathbb{R}^{d}}$ given in Lemma 3.1 then imply

$$
\begin{aligned}
&\left\|f_{H^{1+s}}\right\|_{L^{2}\left(\mathbb{R}^{d} \backslash \Gamma\right)} \leq C\|f\|_{L^{2}\left(\mathbb{R}^{d}\right)} \leq C k^{2}\left\|\widetilde{V}_{0} \varphi\right\|_{L^{2}\left(B_{2 R}\right)}, \\
&\left\|f_{H^{1+s}}\right\|_{H^{t}\left(\mathbb{R}^{d} \backslash \Gamma\right)} \leq C\left(q k^{-1}\right)^{1+s-t} k^{2}\|\varphi\|_{H^{-1 / 2+s}(\Gamma)}, \quad t \in\{0,1+s\}, \\
&\left\|\nabla^{n} f_{\mathcal{A}, p w}\right\|_{L^{2}\left(\mathbb{R}^{d} \backslash \Gamma\right)} \leq C k^{2}(\gamma k)^{n}\left\|\widetilde{V}_{0} \varphi\right\|_{L^{2}\left(B_{2 R}\right)} \quad \forall n \in \mathbb{N}_{0} .
\end{aligned}
$$


It will be advisable to split $f_{H^{1+s}}$ once more, namely, to write

$$
f_{H^{1+s}}=H_{\mathbb{R}^{d}}\left(f_{H^{1+s}}\right)+L_{\mathbb{R}^{d}}\left(f_{H^{1+s}}\right)=: f_{f i n}+f_{\mathcal{A}} .
$$

Since $L_{\mathbb{R}^{d}}\left(f_{H^{1+s}}\right)$ is an entire function and $f_{H^{1+s}}$ is piecewise smooth, we conclude that $f_{\text {fin }}=H_{\mathbb{R}^{d}}\left(f_{H^{1+s}}\right)$ is piecewise smooth. Concerning bounds for $f_{\text {fin }}$, we start by noting that Lemma 3.1 implies

$$
\left\|\nabla^{n} f_{\mathcal{A}}\right\|_{L^{2}\left(\mathbb{R}^{d}\right)} \leq C(\gamma k)^{n}\left\|f_{H^{1+s}}\right\|_{L^{2}\left(\mathbb{R}^{d}\right)} \quad \forall n \in \mathbb{N}_{0} .
$$

Inserting into this the estimates (4.8) and (4.7) leads to two different bounds:

$$
\begin{aligned}
\left\|\nabla^{n} f_{\mathcal{A}}\right\|_{L^{2}\left(\mathbb{R}^{d}\right)} & \leq C(\gamma k)^{n} q^{1+s} k^{1-s}\|\varphi\|_{H^{-1 / 2+s}(\Gamma)} \forall n \in \mathbb{N}_{0}, \\
\left\|\nabla^{n} f_{\mathcal{A}}\right\|_{L^{2}\left(\mathbb{R}^{d}\right)} & \leq C(\gamma k)^{n} k^{2}\left\|\widetilde{V}_{0} \varphi\right\|_{L^{2}\left(B_{2 R}\right)} \quad \forall n \in \mathbb{N}_{0} .
\end{aligned}
$$

The estimate (4.11) together with interpolation inequalities implies

$$
\left\|f_{\mathcal{A}}\right\|_{H^{t}\left(\mathbb{R}^{d}\right)} \lesssim\left(q k^{-1}\right)^{1+s-t} k^{2}\|\varphi\|_{H^{-1 / 2+s}(\Gamma)}, \quad t \in\{0,1+s\} .
$$

The bounds (4.8) and (4.13) imply for $f_{f i n}=f_{H^{1+s}}-f_{\mathcal{A}}$

$$
\begin{aligned}
\left\|f_{f i n}\right\|_{H^{t}\left(\mathbb{R}^{d} \backslash \Gamma\right)} & \lesssim\left\|f_{H^{1+s}}\right\|_{H^{t}\left(\mathbb{R}^{d} \backslash \Gamma\right)}+\left\|f_{\mathcal{A}}\right\|_{H^{t}\left(\mathbb{R}^{d} \backslash \Gamma\right)} \\
& \lesssim\left(q k^{-1}\right)^{1+s-t} k^{2}\|\varphi\|_{H^{-1 / 2+s}(\Gamma)}, \quad t \in\{0,1+s\} .
\end{aligned}
$$

Next, Lemma 3.2 gives for $N_{k}\left(f_{f i n}\right)=N_{k}\left(H_{\mathbb{R}^{d}} f_{H^{1+s}}\right)$

$$
\left\|N_{k}\left(f_{f i n}\right)\right\|_{L^{2}\left(B_{2 R}\right)} \leq C\left(q k^{-1}\right)^{2}\left\|f_{H^{1+s}}\right\|_{L^{2}\left(\mathbb{R}^{d} \backslash \Gamma\right)} \leq C q^{2}\left(q k^{-1}\right)^{1+s}\|\varphi\|_{H^{-1 / 2+s}(\Gamma)}
$$

The regularity theory of Theorem B.6 then implies

$\left\|N_{k}\left(f_{\text {fin }}\right)\right\|_{H^{(s+1)+2}\left(\Omega_{R}\right)} \lesssim$ $k^{s+1}\left\|f_{f i n}\right\|_{L^{2}\left(\mathbb{R}^{d} \backslash \Gamma\right)}+\left\|f_{f i n}\right\|_{H^{1+s}\left(\mathbb{R}^{d} \backslash \Gamma\right)}+k^{(s+1)+2}\left\|N_{k}\left(f_{f i n}\right)\right\|_{L^{2}\left(B_{2 R}\right)} \leq C k^{2}\|\varphi\|_{H^{-1 / 2+s}(\Gamma)}$.

This estimate together with (4.15) can be written as

$$
\left\|N_{k}\left(f_{f i n}\right)\right\|_{H^{t}\left(\Omega_{R}\right)} \lesssim k^{2}\left(q k^{-1}\right)^{3+s-t}\|\varphi\|_{H^{-1 / 2+s}(\Gamma)}, \quad t \in\{0,3+s\} .
$$

The (piecewise) multiplicative interpolation inequality then gives estimates for the intermediate values $0 \leq s^{\prime} \leq 3+s$ :

$$
\begin{aligned}
\left\|N_{k}\left(f_{f i n}\right)\right\|_{H^{s^{\prime}}\left(\Omega_{R}\right)} & \leq C\left\|N_{k}\left(f_{f i n}\right)\right\|_{L^{2}\left(\Omega_{R}\right)}^{\left(3+s-s^{\prime}\right) /(3+s)}\left\|N_{k}\left(f_{f i n}\right)\right\|_{H^{3+s}\left(\Omega_{R}\right)}^{s^{\prime} /(3+s)} \\
& \leq C k^{2}\left(q k^{-1}\right)^{3+s-s^{\prime}}\|\varphi\|_{H^{-1 / 2+s}(\Gamma)} .
\end{aligned}
$$

Upon setting $\left.\widetilde{S}_{V, p w} \varphi:=N_{k}\left(f_{f i n}\right)\right)$ we get the desired estimates for $\widetilde{S}_{V}$. We now turn to the properties of $\mathcal{A}_{V, p w}$, which is defined as $\mathcal{A}_{V, p w} \varphi:=N_{k}\left(f_{\mathcal{A}}\right)+N_{k}\left(f_{\mathcal{A}, p w}\right)$. Lemma 3.2 implies

$$
\begin{aligned}
& \sum_{j=0}^{1} k^{-j}\left\|N_{k}\left(f_{\mathcal{A}, p w}\right)\right\|_{H^{j}\left(B_{2 R}\right)}+\sum_{j=0}^{1} k^{-j}\left\|N_{k}\left(f_{\mathcal{A}}\right)\right\|_{H^{j}\left(B_{2 R}\right)} \\
& \lesssim k^{-1}\left\|f_{\mathcal{A}}\right\|_{L^{2}\left(\mathbb{R}^{d}\right)}+k^{-1}\left\|f_{\mathcal{A}, p w}\right\|_{L^{2}\left(\mathbb{R}^{d}\right)} \leq C k\left\|\widetilde{V}_{0} \varphi\right\|_{L^{2}\left(B_{2 R}\right)} . \\
& 11
\end{aligned}
$$


(4.16) and Theorem B.4 produce

$\left\|\nabla^{n+2} \widetilde{\mathcal{A}}_{V, p w} \varphi\right\|_{L^{2}\left(\Omega_{R}\right)} \leq C \max \{n, k\}^{n+2} \gamma^{n}\left[\left\|\widetilde{V}_{0} \varphi\right\|_{L^{2}\left(B_{2 R}\right)}+k\left\|\widetilde{V}_{0} \varphi\right\|_{L^{2}\left(B_{2 R}\right)}\right] \quad \forall n \in \mathbb{N}_{0}$

for suitable constants $C, \gamma>0$ independent of $n$ and $k$. Together with (4.16) and the observation $\left\|\widetilde{V}_{0} \varphi\right\|_{L^{2}\left(\Omega_{R}\right)} \leq C\|\varphi\|_{H^{-3 / 2}(\Gamma)}$ (cf. Lemma 2.4) this implies the desired estimates for $\widetilde{A}_{V, p w} \varphi$. $\mathrm{\square}$

The proof of Theorem 4.3 relies on two facts, namely, on a piecewise shift theorem for $\widetilde{V}_{0}$ and regularity theory for Helmholtz transmission problems. The same arguments can therefore be used for the double layer potential $\widetilde{K}$.

Theorem 4.4 (decomposition of $\widetilde{K}$, analytic boundary). Let $\Gamma$ be analytic and $q \in(0,1)$. Then we can decompose $\widetilde{K}$ as

$$
\widetilde{K}=\widetilde{K}_{0}+\widetilde{S}_{K, p w}+\widetilde{\mathcal{A}}_{K, p w}
$$

such that for every $s \geq-1$ :

(i) $\widetilde{S}_{K, p w}: H^{1 / 2+s}(\bar{\Gamma}) \rightarrow H^{2}\left(B_{R}\right) \cap H^{3+s}\left(\Omega_{R}\right)$ with

$$
\left\|\widetilde{S}_{K, p w} \varphi\right\|_{H^{s^{\prime}\left(\Omega_{R}\right)}} \leq C_{s^{\prime}, s} q^{2}\left(q k^{-1}\right)^{1+s-s^{\prime}}\|\varphi\|_{H^{1 / 2+s}(\Gamma)}, \quad 0 \leq s^{\prime} \leq s+3
$$

Here, the constant $C_{s^{\prime}, s}>0$ is independent of $q$ and $k \geq k_{0}$.

(ii) $\widetilde{\mathcal{A}}_{K, p w}: H^{1 / 2+s}(\Gamma) \rightarrow H^{2}\left(B_{R}\right)$ maps into a space of piecewise analytic functions and

$$
\left\|\nabla^{n} \widetilde{\mathcal{A}}_{K, p w} \varphi\right\|_{L^{2}\left(\Omega_{R}\right)} \leq C k \gamma^{n} \max \{n+1, k\}^{n}\|\varphi\|_{H^{-1 / 2}(\Gamma)} \quad \forall n \in \mathbb{N}_{0} .
$$

Here, the constants $C, \gamma>0$ are independent of $k \geq k_{0}$ but may depend on $q$. Proof. The proof is analogous to that of Theorem 4.3.

4.2.1. further mapping properties of the operators $\widetilde{V}$ and $\widetilde{K}$. The results of Section 4.2 permit us to formulate the following corollary.

COROLlary 4.5. Let $\Gamma$ be analytic. Then

$$
\begin{aligned}
\|\widetilde{V} \varphi\|_{L^{2}\left(\Omega_{R}\right)} & \leq C k\|\varphi\|_{H^{-3 / 2}(\Gamma)}, \\
\|\widetilde{V} \varphi\|_{H^{1}\left(\Omega_{R}\right)} & \leq C\left[\|\varphi\|_{H^{-1 / 2}(\Gamma)}+k^{2}\|\varphi\|_{H^{-3 / 2}(\Gamma)}\right], \\
\|\widetilde{K} \varphi\|_{L^{2}\left(\Omega_{R}\right)} & \leq C k\|\varphi\|_{H^{-1 / 2}(\Gamma)}, \\
\|\widetilde{K} \varphi\|_{H^{1}\left(\Omega_{R}\right)} & \leq C\left[\|\varphi\|_{H^{1 / 2}(\Gamma)}+k^{2}\|\varphi\|_{H^{-1 / 2}(\Gamma)}\right], \\
k^{2}\|\widetilde{V} \varphi\|_{H^{-1}\left(B_{R}\right)} & \leq C k^{2}\|\varphi\|_{H^{-3 / 2}(\Gamma)}, \\
k^{2}\|\widetilde{K} \varphi\|_{H^{-1}\left(B_{R}\right)} & \leq C k^{2}\|\varphi\|_{H^{-1 / 2}(\Gamma)} .
\end{aligned}
$$

Furthermore, since for $\varphi \in H^{-1 / 2}(\Gamma)$ we have $\widetilde{V} \varphi, \widetilde{K} \varphi \in L^{2}\left(B_{R}\right)$, there holds for every open subset $\omega \subset B_{R}$ :

$$
\|\widetilde{V} \varphi\|_{H^{-1}(\omega)} \leq\|\widetilde{V} \varphi\|_{H^{-1}\left(B_{R}\right)}, \quad\|\widetilde{K} \varphi\|_{H^{-1}(\omega)} \leq\|\widetilde{K} \varphi\|_{H^{-1}\left(B_{R}\right)} .
$$

Proof. For the $L^{2}$ - and $H^{1}$-bounds, combine Theorems 4.3, 4.4 with Lemma 2.3. For the $H^{-1}$-estimates, we proceed as follows. For the double layer potential $\widetilde{K} \varphi \in$ $L^{2}\left(\Omega_{R}\right)$ we use the differential equation to get for $v \in H_{0}^{1}\left(B_{R}\right)$

$$
k^{2}\langle\widetilde{K} \varphi, v\rangle=-\int_{\Omega_{R}} \Delta \widetilde{K} \varphi v=-\int_{\Omega_{R}} \Delta\left(\widetilde{S}_{K} \varphi+\widetilde{\mathcal{A}}_{K} \varphi\right) v .
$$


An integration by parts and the observations that $\widetilde{S}_{K} \varphi$ and $\widetilde{\mathcal{A}}_{K} \varphi \in H^{2}\left(B_{R}\right)$ (and thus their normal derivative does not jump across $\Gamma$ ) yield together with Theorem 4.4

$$
\begin{aligned}
\left|k^{2}\langle\widetilde{K} \varphi, v\rangle\right| & =\left|\int_{\Omega_{R}} \nabla\left(\widetilde{S}_{K} \varphi+\widetilde{\mathcal{A}}_{K} \varphi\right) \cdot \nabla v\right| \\
& \leq C\left[q^{2}\left(q k^{-1}\right)^{s}\|\varphi\|_{H^{1 / 2+s}(\Gamma)}+k^{2}\|\varphi\|_{H^{-1 / 2}(\Gamma)}\right]\|\nabla v\|_{L^{2}\left(B_{R}\right)} .
\end{aligned}
$$

Selecting $s=-1$ leads to the claim estimate. For $\|\widetilde{V} \varphi\|_{H^{-1}\left(B_{R}\right)}$, we proceed analogously. [

For later reference, we collect some interior regularity results for solutions to the homogeneous Helmholtz equation.

LEMMA 4.6. Let $\omega^{\prime} \subset \subset \omega \subset \mathbb{R}^{d}$ be two bounded Lipschitz domains. Let $u \in L^{2}(\omega)$ solve the homogeneous Helmholtz equation. Then there exists $C>0$ (depending only on $\operatorname{dist}\left(\omega^{\prime}, \partial \omega\right)>0, \omega$, and $\left.k_{0}\right)$ such that

$$
\|u\|_{\mathcal{H}, \omega^{\prime}} \leq C k^{2}\|u\|_{H^{-1}(\omega)} .
$$

If $u \in H^{1}(\omega)$, then we have

$$
\left\|\partial_{n} u\right\|_{H^{-1 / 2}(\omega)} \leq C k\|u\|_{\mathcal{H}, \omega} .
$$

Proof. For every smooth cut-off function $\chi$ with $\operatorname{supp} \chi \subset \omega$ we have $\|\chi u\|_{H^{-1}(\omega)} \leq$ $C\|u\|_{H^{-1}(\omega)}$. Next, classical interior regularity gives us

$$
\|\nabla u\|_{L^{2}\left(\omega^{\prime}\right)} \leq C k^{2}\|u\|_{L^{2}\left(\omega^{\prime \prime}\right)}
$$

for all $\omega^{\prime} \subset \subset \omega^{\prime \prime} \subset \subset \omega$ Next, to get the $L^{2}$-estimate we observe that $\chi u$ satisfies

$$
-\Delta(\chi u)+k^{2} \chi u=2 k^{2} \chi u-2 \nabla \chi \cdot \nabla u-\Delta \chi u, \quad \chi u=0 \quad \text { on } \partial \omega .
$$

Lax-Milgram for the operator $-\Delta+k^{2}$ Id then gives

$$
\|\chi u\|_{\mathcal{H}, \omega} \leq C k^{2}\|\chi u\|_{H^{-1}(\omega)} \leq C k^{2}\|u\|_{H^{-1}(\omega)} .
$$

We now turn to the case of $u \in H^{1}(\omega)$. For $v \in H^{1}(\omega)$ we have

$$
\left|\left\langle\partial_{n} u, v\right\rangle\right|=\left|\int_{\omega} \nabla u \cdot \nabla v+\int_{\omega} \Delta u v\right|=\left|\int_{\omega} \nabla u \cdot \nabla v-k^{2} \int_{\omega} u v\right| \leq\|u\|_{\mathcal{H}, \omega}\|v\|_{\mathcal{H}, \omega}
$$

which implies the stated estimate.

5. decomposition of combined field operators. The combined field operators $A$ and $A^{\prime}$ of (1.3), (1.4) are linear combinations of the operators $V$ and $K$. Hence, the decompositions of the operators $V$ and $K$ of Section 4 imply decompositions of $A$ and $A^{\prime}$. The purpose of the present section is to give these decompositions a form that will be convenient later on. We restrict our attention to the case of analytic boundaries $\Gamma$.

5.1. frequency splitting for function spaces on surfaces and domains. An important tool for the analysis will the "frequency splitting" operators analogous to the operators $H_{\mathbb{R}^{d}}$ and $L_{\mathbb{R}^{d}}$ of Lemma 3.1. We have

LEMMA 5.1 (frequency splitting on domains). Let $q \in(0,1)$ and $\Omega$ be a bounded Lipschitz domain. Then one can construct operators $L_{\Omega}$ and $H_{\Omega}$ defined on $L^{2}(\Omega)$ with the following properties: 
(i) $H_{\Omega}+L_{\Omega}=\mathrm{Id}$

(ii) $\left\|H_{\Omega} f\right\|_{H^{s^{\prime}(\Omega)}} \leq C_{s, s^{\prime}}\left(q k^{-1}\right)^{s-s^{\prime}}\|f\|_{H^{s}(\Omega)}$, where $0 \leq s^{\prime} \leq s$ and $s \geq 0$.

(iii) $L_{\Omega} f$ is an entire function on $\mathbb{R}^{d}$ and

$$
\left\|\nabla^{n} L_{\Omega} f\right\|_{L^{2}\left(\mathbb{R}^{d}\right)} \leq C(\gamma k)^{n}\|f\|_{L^{2}(\Omega)} \quad \forall n \in \mathbb{N}_{0} .
$$

Here, $C_{s, s^{\prime}}$ is independent of $k$ and $q$; the constants $C, \gamma$ are independent of $k$.

Proof. Let $E_{\Omega}: L^{2}(\Omega) \rightarrow L^{2}\left(\mathbb{R}^{d}\right)$ be the Stein extension operator. Then define $H_{\Omega} f=\left.\left(H_{\mathbb{R}^{d}} \circ E_{\Omega} f\right)\right|_{\Omega}$ and $L_{\Omega} f:=\left(L_{\mathbb{R}^{d}} \circ E_{\Omega} f\right)$. The properties then follow from Lemma 3.1.

LEMma 5.2 (frequency splitting on surfaces). Let $\Omega \subset \mathbb{R}^{d}$ be a bounded Lipschitz domain with smooth boundary $\Gamma$. Let $s>0$ and $q \in(0,1)$. Then one can construct operators $L_{\Gamma}: H^{s}(\Gamma) \rightarrow H^{1 / 2+s}\left(\mathbb{R}^{d}\right)$ and $H_{\Gamma}: H^{s}(\Gamma) \rightarrow H^{s}(\Gamma)$ with the following properties:

(i) $H_{\Gamma}+\gamma_{0}^{i n t} L_{\Gamma}=\mathrm{Id}$

(ii) $\left\|H_{\Gamma} f\right\|_{H^{s^{\prime}(\Gamma)}} \leq C_{s, s^{\prime}}\left(q k^{-1}\right)^{s-s^{\prime}}\|f\|_{H^{s}(\Gamma)}$, where $0 \leq s^{\prime} \leq s$.

(iii) $L_{\Gamma} f$ is an entire function on $\mathbb{R}^{d}$ and

$$
\left\|\nabla^{n} L_{\Gamma} f\right\|_{L^{2}\left(\mathbb{R}^{d}\right)} \leq C(\gamma k)^{n-(1 / 2+s)}\|f\|_{H^{s}(\Gamma)} \quad \forall n \in \mathbb{N}_{0} .
$$

Here, the constant $C_{s, s^{\prime}}$ is independent of $k$ and $q$; the constants $C, \gamma$ are independent of $k$.

Proof. Related frequency splittings have been constructed in [21]. We therefore merely sketch the construction. Let $G: H^{s}(\Gamma) \rightarrow H^{1 / 2+s}\left(\mathbb{R}^{d}\right)$ be a lifting operator. Define $H_{\Gamma}:=\gamma_{0}^{\text {int }} \circ H_{\mathbb{R}^{d}} \circ G$ and $L_{\Gamma}:=L_{\mathbb{R}^{d}} \circ G$. The properties of $H_{\mathbb{R}^{d}}$ and $L_{\mathbb{R}^{d}}$ given Lemma 3.1 then imply the statements. For example, the bound for $H_{\Gamma}$ follows from the properties of $H_{\mathbb{R}^{d}}$. Specifically, the multiplicative trace inequality (see, e.g., [20, Thm. A.2]) yields

$$
\begin{aligned}
\left\|H_{\Gamma} \varphi\right\|_{L^{2}(\Gamma)} & \leq\left\|H_{\mathbb{R}^{d}}(G \varphi)\right\|_{L^{2}(\Omega)}^{2 s /(1+2 s)}\left\|H_{\mathbb{R}^{d}}(G \varphi)\right\|_{H^{1 / 2+s}(\Omega)}^{1 /(1+2 s)} \\
& \lesssim\left(q k^{-1}\right)^{s}\|G \varphi\|_{H^{1 / 2+s}(\Omega)} \lesssim\left(q k^{-1}\right)^{s}\|\varphi\|_{H^{s}(\Gamma)}
\end{aligned}
$$

on the other hand, trace inequalities and the stability of $H_{\mathbb{R}^{d}}$ yield

$$
\left\|H_{\Gamma} \varphi\right\|_{H^{s}(\Gamma)} \lesssim\left\|H_{\mathbb{R}^{d}}(G \varphi)\right\|_{H^{1 / 2+s}(\Omega)} \lesssim\|G \varphi\|_{H^{1 / 2+s}(\Omega)} \lesssim\|\varphi\|_{H^{s}(\Gamma)} .
$$

Thus, the limiting cases $s^{\prime} \in\{0, s\}$ are proved. The intermediate cases $0<s^{\prime}<s$ follow by interpolation arguments.

The frequency splitting in Lemma 5.2 relies on a frequency splitting in a domain and the trace operator. This precludes a direct extension of the construction to negativeindex Sobolev spaces. Nevertheless, splittings can be defined on such spaces, and the following lemma presents one possible construction.

LEMMA 5.3 (frequency splitting on surfaces, negative norms). Let $\Omega \subset \mathbb{R}^{d}$ be a bounded Lipschitz domain with an analytic boundary $\Gamma$. Let $q \in(0,1)$. Then one can construct operators $L_{\Gamma}^{\text {neg }}, H_{\Gamma}^{\text {neg }}$ on $H^{-1}(\Gamma)$ with the following properties:

(i) $L_{\Gamma}^{\text {neg }}+H_{\Gamma}^{\text {neg }}=\mathrm{Id}$

(ii) for $-1 \leq s^{\prime} \leq s \leq 1$ :

$$
\left\|H_{\Gamma}^{\text {neg }} f\right\|_{H^{s^{\prime}(\Gamma)}} \leq C(q / k)^{s-s^{\prime}}\|f\|_{H^{s}(\Gamma)}
$$


(iii) $L_{\Gamma}^{n e g} f$ is the restriction to $\Gamma$ of a function that is analytic on a tubular neighborhood $T$ of $\Gamma$ and satisfies

$$
\left\|\nabla^{n} L_{\Gamma}^{n e g} f\right\|_{L^{2}(T)} \leq C k^{d / 2} \gamma^{n} \max \{k, n\}^{n}\|f\|_{H^{-1 / 2}(\Gamma)} \quad \forall n \in \mathbb{N}_{0} .
$$

Proof. Consider on the compact manifold $\Gamma$ for the Laplace-Beltrami operator $\Delta_{\Gamma}$ the eigenvalue problem

$$
-\Delta_{\Gamma} \varphi-\lambda^{2} \varphi=0 \quad \text { on } \Gamma .
$$

There are countably many eigenfunctions $\varphi_{m}, m \in \mathbb{N}_{0}$, with associated eigenvalues $\lambda_{m} \geq 0$, which we assume to be sorted in ascending order. Without loss of generality, we impose the normalization $\left\|\varphi_{m}\right\|_{L^{2}(\Gamma)}=1$. We have Weyl's formula (see [8, p. 155])

$$
N(\lambda):=\operatorname{card}\left\{\lambda_{m} \mid \lambda_{m} \leq \lambda\right\} \sim C_{\Gamma} \lambda^{d-1},
$$

where the constant $C_{\Gamma}$ depends solely on $\Gamma$. Additionally, we have from Lemma C.1 the existence of a tubular neighborhood $T$ of $\Gamma$ and constants $C, \gamma>0$ such that

$$
\left\|\nabla^{n} \varphi_{m}\right\|_{L^{2}(T)} \leq C \gamma^{n}\left\{\lambda_{m}, n\right\}^{n} \quad \forall n \in \mathbb{N}_{0} .
$$

Furthermore, the functions $\left(\varphi_{m}\right)_{m=0}^{\infty}$ are an orthonormal basis of $L^{2}(\Gamma)$ and an orthogonal basis of $H^{1}(\Gamma)$ :

$$
\begin{aligned}
& \|u\|_{L^{2}(\Gamma)}^{2}=\sum_{m=0}^{\infty}\left|\left\langle u, \varphi_{m}\right\rangle_{L^{2}(\Gamma)}\right|^{2} \quad \forall u \in L^{2}(\Gamma), \\
& \|u\|_{H^{1}(\Gamma)}^{2}=\sum_{m=0}^{\infty}\left(1+\lambda_{m}^{2}\right)\left|\left\langle u, \varphi_{m}\right\rangle_{L^{2}(\Gamma)}\right|^{2} \quad \forall u \in H^{1}(\Gamma) .
\end{aligned}
$$

By interpolation, we get for $0 \leq s \leq 1$ and $u \in L^{2}(\Gamma)$ :

$$
\|u\|_{H^{s}(\Gamma)}^{2} \sim \sum_{m=0}^{\infty}\left(1+\lambda_{m}^{2}\right)^{s}\left|\left\langle u, \varphi_{m}\right\rangle_{L^{2}(\Gamma)}\right|^{2} .
$$

By duality, distributions $f \in H^{s}(\Gamma)$ with $s \in[-1,0]$ can be identified with sequences $\left(f_{m}\right)_{m=0}^{\infty}$ such that $\|f\|_{H^{s}(\Gamma)}^{2} \sim \sum_{m=0}^{\infty}\left(1+\lambda_{m}^{2}\right)^{s}\left|f_{m}\right|^{2}$. We will write (formally) $f=$ $\sum_{m=0}^{\infty} f_{m} \varphi_{m}$ to express this identification.

We now define the operators $H_{\Gamma}^{\text {neg }}$ and $L_{\Gamma}^{\text {neg }}$ by

$$
H_{\Gamma}^{\text {neg }} f:=\sum_{m: \lambda_{m}>\eta k} f_{m} \varphi_{m}, \quad L_{\Gamma}^{n e g} f:=\sum_{m: \lambda_{m} \leq \eta k} f_{m} \varphi_{m}
$$

Then clearly $H_{\Gamma}^{\text {neg }}+L_{\Gamma}^{\text {neg }}=\mathrm{Id}$. Next, in the tubular neighborhood $T$ of $\Gamma$ we have

$$
\begin{aligned}
\left\|\nabla^{n} L_{\Gamma}^{n e g} f\right\|_{L^{2}(T)} & \leq \sum_{m: \lambda_{m} \leq \eta k}\left|f_{m}\right|\left\|\nabla^{n} \varphi_{m}\right\|_{L^{2}(T)} \leq C \gamma^{n} \max \{\eta k, n\}^{n} \sum_{m: \lambda_{m} \leq \eta k}\left|f_{m}\right| \\
& \leq C \gamma^{n} \max \{\eta k, n\}^{n} \sqrt{\sum_{m: \lambda_{m} \leq \eta k}\left(1+\lambda_{m}^{2}\right)^{1 / 2}} \sqrt{\sum_{m: \lambda_{m} \leq \eta k}\left(1+\lambda_{m}^{2}\right)^{-1 / 2}\left|f_{m}\right|^{2}} \\
& \leq C \gamma^{n} \max \{\eta k, n\}^{n}\left(1+(\eta k)^{2}\right)^{1 / 4} \sqrt{N(\eta k)}\|f\|_{H^{-1 / 2}(\Gamma)} \\
& \leq C k^{d / 2} \gamma^{n} \max \{\eta k, n\}^{n}\|f\|_{H^{-1 / 2}(\Gamma)} .
\end{aligned}
$$


For $H_{\Gamma}^{\text {neg }} f$, we compute for $-1 \leq s^{\prime} \leq s \leq 1$ :

$$
\left\|H_{\Gamma}^{n e g} f\right\|_{H^{s^{\prime}}(\Gamma)}^{2} \leq C \sum_{m: \lambda_{m}>\eta k}\left(1+\lambda_{m}\right)^{2 s^{\prime}}\left|f_{m}\right|^{2} \leq C(1+\eta k)^{2\left(s^{\prime}-s\right)}\|f\|_{H^{s}(\Gamma)}^{2},
$$

which finishes the proof. $\square$

REMARK 5.4. The factor $k^{d / 2}$ in the estimates for $L_{\Gamma}^{n e g}$ is not optimal and can be reduced (see Remark C.2). Also, the proof shows that the term $\|f\|_{H^{-1 / 2}(\Gamma)}$ in the bounds for $L_{\Gamma}^{n e g}$ can be reduced to $\|f\|_{H^{-1}(\Gamma)}$ at the expense of further powers of $k$.

5.2. decomposition of $A$ and $A^{\prime}$. We recall the definition of $\mathfrak{A}(C, \gamma, T \backslash \Gamma)$ given in Definition 1.1 and the definition of the jump operator [.] in Section 1.1.1. Lemma 5.5 (decomposition of $A$ ). Let $\Gamma$ be analytic and let $s \geq 0$. Fix $q \in(0,1)$. Then the operator $A$ can be written as

$$
A= \pm \frac{1}{2}+K_{0}+R_{A}+k\left[\widetilde{\mathcal{A}}_{A}\right]
$$

where $R_{A}: H^{s}(\Gamma) \rightarrow H^{s+1}(\Gamma)$ and $\widetilde{\mathcal{A}}_{A}$ satisfy for some constant $C$, which is independent of $k \geq k_{0}$ and $q$, and a constant $\gamma>0$, which is independent of $k \geq k_{0}$,

$$
\begin{aligned}
& \left\|R_{A}\right\|_{H^{s+1}(\Gamma) \leftarrow H^{s}(\Gamma)} \leq C k, \quad\left\|R_{A}\right\|_{H^{s}(\Gamma) \leftarrow H^{s}(\Gamma)} \leq q, \\
& \left.\widetilde{\mathcal{A}}_{A} \varphi \in \mathfrak{A}\left(C C_{\varphi}, \gamma, \Omega_{R}\right)\right), \quad C_{\varphi}=\|\varphi\|_{H^{-1 / 2}(\Gamma)}+k\|\varphi\|_{H^{-3 / 2}(\Gamma)} .
\end{aligned}
$$

Proof. Before turning to the proof, we point out that, since only the jump of the potential $\widetilde{A}_{A} \varphi$ across $\Gamma$ appears in the decomposition of $A$, there is some freedom in the choice of $\widetilde{A}_{A}$. In particular, $\widetilde{\mathcal{A}}_{A}$ can be selected such that $\left.\left(\widetilde{\mathcal{A}}_{A} f\right)\right|_{\Omega^{+}}=0$ or $\left.\left(\widetilde{\mathcal{A}}_{A} f\right)\right|_{\Omega}=0$. Indeed, we will construct $\widetilde{\mathcal{A}}_{A}$ such that $\widetilde{\mathcal{A}}_{A} f=0$ on $\Omega^{+}$if $A=$ $-1 / 2+K-\mathbf{i} \eta V$ is considered and $\widetilde{\mathcal{A}}_{A} f=0$ on $\Omega$ if $A=1 / 2+K-\mathbf{i} \eta V$.

We will only consider the operator $A$ given in (1.3a) (i.e., the case $A=-1 / 2+$ $K-\mathbf{i} \eta V)$, the other case being handled analogously. Since $A=\gamma_{0}^{i n t}(\widetilde{K}-\mathbf{i} \eta \widetilde{V})$, the decompositions of $\widetilde{K}$ and $\widetilde{V}$ of Theorems $4.4,4.3$ produce

$$
A=\left\{-\frac{1}{2}+K_{0}\right\}+\left\{\gamma_{0}^{i n t}\left(\widetilde{S}_{K, p w}-\mathbf{i} \eta \widetilde{S}_{V, p w}\right)-\mathbf{i} \eta V_{0}\right\}+\left\{\gamma_{0}^{i n t}\left(\widetilde{\mathcal{A}}_{K, p w}-\mathbf{i} \eta \widetilde{\mathcal{A}}_{V, p w}\right)\right\} .
$$

With the aid of the high and low frequency operators $H_{\Gamma}$ and $L_{\Gamma}$ of Lemma 5.2, we write $V_{0}=H_{\Gamma} V_{0}+\gamma_{0}^{\text {int }} L_{\Gamma} V_{0}$ and therefore arrive at the decomposition

$$
\begin{aligned}
A & =-1 / 2+K_{0}+R_{A}+\gamma_{0}^{i n t} \widetilde{\mathcal{A}}_{A}, \\
R_{A} & =\gamma_{0}^{i n t}\left(\widetilde{S}_{K, p w}-\mathbf{i} \eta \widetilde{S}_{V, p w}\right)-\mathbf{i} H_{\Gamma} \eta V_{0}, \\
\widetilde{\mathcal{A}}_{A} & =-\mathbf{i} \eta k^{-1} L_{\Gamma} V_{0}+k^{-1} \widetilde{\mathcal{A}}_{K, p w}-\mathbf{i} \eta k^{-1} \widetilde{\mathcal{A}}_{V, p w} .
\end{aligned}
$$

It remains to obtain the stated bounds. Theorems 4.3, 4.4 and Lemma 5.2 produce (for notational convenience, we employ the same parameter $q \in(0,1)$ in the splittings of Theorems 4.3, 4.4 and Lemma 5.2)

$$
\begin{aligned}
\left\|\gamma_{0}^{i n t} \widetilde{S}_{V, p w}\right\|_{H^{1+s}(\Gamma) \leftarrow H^{s}(\Gamma)} & \leq C q^{2}, & \left\|\gamma_{0}^{i n t} \widetilde{S}_{V, p w}\right\|_{H^{s}(\Gamma) \leftarrow H^{s}(\Gamma)} & \leq C q^{3} k^{-1}, \\
\left\|\gamma_{0}^{i n t} \widetilde{S}_{K, p w}\right\|_{H^{1+s}(\Gamma) \leftarrow H^{s}(\Gamma)} & \leq C q k, & \left\|\gamma_{0}^{i n t} \widetilde{S}_{K, p w}\right\|_{H^{s}(\Gamma) \leftarrow H^{s}(\Gamma)} & \leq C q^{2}, \\
\left\|H_{\Gamma} V_{0}\right\|_{H^{1+s}(\Gamma) \leftarrow H^{s}(\Gamma)} & \leq C, & \left\|H_{\Gamma} V_{0}\right\|_{H^{s}(\Gamma) \leftarrow H^{s}(\Gamma)} & \leq C q k^{-1} .
\end{aligned}
$$


By selecting $q$ sufficiently small, we can obtain the desired bounds for $R_{A}$. For $\widetilde{\mathcal{A}}_{A}$ we see that Theorems 4.3, 4.4, and Lemma 5.2 together with the mapping properties of $V_{0}$ yield

$$
\varphi \in H^{-1 / 2}(\Gamma) \Longrightarrow \widetilde{\mathcal{A}}_{A} \varphi \in \mathfrak{A}\left(C C_{\varphi}, \gamma, \Omega_{R}\right), \quad C_{\varphi}:=\|\varphi\|_{H^{-1 / 2}(\Gamma)}+k\|\varphi\|_{H^{-3 / 2}(\Gamma)} .
$$

This concludes the proof.

REMARK 5.6. The operator $-1 / 2+K_{0}$ is invertible while the operator $1 / 2+K_{0}$ has a one-dimensional kernel. It is convenient to have decompositions with invertible leading term. By Lemma 2.5, the operator $1 / 2+K_{0}-\mathbf{i} V_{0}$ is invertible. Inspection of the proof of Lemma 5.5 shows that we can achieve a decomposition of the following form:

$$
1 / 2+K-\mathbf{i} \eta V=\frac{1}{2}+K_{0}+\mathbf{i} V_{0}+R_{A}+k\left[\widetilde{\mathcal{A}}_{A}\right]
$$

where the operators $R_{A}$ and $\widetilde{\mathcal{A}}_{A}$ have the regularity properties stated in Lemma 5.5.

The next two lemmas provide decompositions of $A^{\prime}$ - the difference between these two results lies in the range of Sobolev spaces on which they are defined: While Lemma 5.7 covers the case $s \geq 0$, Lemma 5.9 extends the range to $s \geq-1 / 2$ at the expense of further powers of $k$.

Lemma 5.7 (decomposition of $A^{\prime}$ ). Let $\Gamma$ be analytic and let $s \geq 0$. Fix $q \in(0,1)$. Then the operator $A^{\prime}$ can be written in the form

$$
A^{\prime}= \pm \frac{1}{2}+K_{0}^{\prime}+R_{A^{\prime}}+k\left[\widetilde{\mathcal{A}}_{A^{\prime}, 1}\right]+\left[\partial_{n} \widetilde{\mathcal{A}}_{A^{\prime}, 2}\right]
$$

where $R_{A^{\prime}}: H^{s}(\Gamma) \rightarrow H^{s+1}(\Gamma)$ and $\widetilde{\mathcal{A}}_{A^{\prime}}$ satisfy for some constants $C, \gamma>0$ that are independent of $k \geq k_{0}$

$$
\begin{aligned}
& \left\|R_{A^{\prime}}\right\|_{H^{s+1}(\Gamma) \leftarrow H^{s}(\Gamma)} \leq C k, \quad\left\|R_{A^{\prime}}\right\|_{H^{s}(\Gamma) \leftarrow H^{s}(\Gamma)} \leq q, \\
& \left.\widetilde{\mathcal{A}}_{A^{\prime}, i} \varphi \in \mathfrak{A}\left(C C_{\varphi}, \gamma, \Omega_{R}\right)\right), \quad C_{\varphi}=k\|\varphi\|_{H^{-3 / 2}(\Gamma)}, \quad i \in\{1,2\} .
\end{aligned}
$$

Proof. We consider the case $A^{\prime}=\frac{1}{2}+K^{\prime}+\mathbf{i} \eta V$, the case $A^{\prime}=-1 / 2+K^{\prime}+\mathbf{i} \eta V$ being handled by analogous arguments. We recall that the operator $A^{\prime}$ is given by $A^{\prime} \varphi=\gamma_{1}^{i n t} \widetilde{V} \varphi-\mathbf{i} \eta \gamma_{0}^{i n t} \widetilde{V} \varphi$. In view of $\gamma_{1}^{i n t} \widetilde{V}_{0}=1 / 2+K_{0}^{\prime}$ we can write with the decomposition of Theorem 4.3

$$
A^{\prime}=\frac{1}{2}+K_{0}^{\prime}+\gamma_{1}^{i n t}\left(\widetilde{S}_{V, p w}+\widetilde{\mathcal{A}}_{V, p w}\right)+\mathbf{i} \eta \gamma_{0}^{i n t}\left(\widetilde{V}_{0}+\widetilde{S}_{V, p w}+\widetilde{\mathcal{A}}_{V, p w}\right) .
$$

Here, the parameter $q$ appearing in the definition of the decomposition of Theorem 4.3 is still at our disposal. Using the high and low frequency operators $H_{\Omega}$ of $L_{\Omega}$ (the parameter $q$ appearing in their definition will be selected shortly) we can set

$$
\begin{aligned}
R_{A^{\prime}} & =\gamma_{1}^{i n t} \widetilde{S}_{V, p w}+\mathbf{i} \eta \gamma_{0}^{i n t} \widetilde{S}_{V, p w}+\mathbf{i} \eta \gamma_{0}^{i n t} H_{\Omega} \widetilde{V}_{0}, \\
\widetilde{\mathcal{A}}_{A^{\prime}, 1} & =-k^{-1} \chi_{\Omega}\left(\mathbf{i} \eta \widetilde{\mathcal{A}}_{V, p w}+\mathbf{i} \eta L_{\Omega} \widetilde{V}_{0}\right), \\
\widetilde{\mathcal{A}}_{A^{\prime}, 2} & =-\chi_{\Omega} \widetilde{\mathcal{A}}_{V, p w}, \quad
\end{aligned}
$$


where $\chi_{\Omega}$ denotes the characteristic function for $\Omega$. Theorem 4.3 yields

$$
\begin{array}{ll}
\left\|\gamma_{1}^{i n t} \widetilde{S}_{V, p w}\right\|_{H^{1+s}(\Gamma) \leftarrow H^{s}(\Gamma)} \leq C q k, & \left\|\gamma_{1}^{i n t} \widetilde{S}_{V, p w}\right\|_{H^{s}(\Gamma) \leftarrow H^{s}(\Gamma)} \leq C q^{2} \\
\left\|\gamma_{0}^{i n t} \widetilde{S}_{V, p w}\right\|_{H^{1+s}(\Gamma) \leftarrow H^{s}(\Gamma)} \leq C q^{2}, & \left\|\gamma_{0}^{i n t} \widetilde{S}_{V, p w}\right\|_{H^{s}(\Gamma) \leftarrow H^{s}(\Gamma)} \leq C q^{3} k^{-1} \\
\left\|\gamma_{0}^{i n t} H_{\Omega} \widetilde{V}_{0}\right\|_{H^{1+s}(\Gamma) \leftarrow H^{s}(\Gamma)} \leq C, & \left\|\gamma_{0}^{i n t} H_{\Omega} \widetilde{V}_{0}\right\|_{H^{s}(\Gamma) \leftarrow H^{s}(\Gamma)} \leq C q k^{-1} .
\end{array}
$$

Selecting $q$ appropriately gives the desired bounds for $R_{A^{\prime}}$. From Theorem 4.3, Lemma 5.1, and Lemma 2.4 we infer

$$
\widetilde{\mathcal{A}}_{A^{\prime}, 2} \varphi \quad \text { and } \quad \widetilde{\mathcal{A}}_{A^{\prime}, 1} \varphi \in \mathfrak{A}\left(C C_{\varphi}, \gamma, \Omega_{R}\right), \quad C_{\varphi}:=k\|\varphi\|_{H^{-3 / 2}(\Gamma)} .
$$

\section{$\square$}

REMARK 5.8. The operator $-1 / 2+K_{0}^{\prime}$ is invertible while the operator $1 / 2+K_{0}^{\prime}$ has a one-dimensional kernel. By Lemma 2.5, the operator $1 / 2+K_{0}^{\prime}+\mathbf{i} V_{0}$ is invertible. Inspection of the proof of Lemma 5.7 shows that we can achieve a decomposition of the following form:

$$
1 / 2+K^{\prime}+\mathbf{i} \eta V=\frac{1}{2}+K_{0}^{\prime}-\mathbf{i} V_{0}+R_{A}+k\left[\widetilde{\mathcal{A}}_{A^{\prime}, 1}\right]+\left[\partial_{n} \widetilde{\mathcal{A}}_{A^{\prime}, 2}\right],
$$

where the operators $R_{A}$ and $\widetilde{\mathcal{A}}_{A^{\prime}, i}, i \in\{1,2\}$ have the regularity properties stated in Lemma 5.7.

Lemma 5.9 (decomposition of $A^{\prime}$ ). Let $\Gamma$ be analytic and let $-1 / 2 \leq s \leq 0$. Fix $q \in(0,1)$. Then the operator $A^{\prime}$ can be written in the form

$$
A^{\prime}= \pm \frac{1}{2}+K_{0}^{\prime}+R_{A^{\prime}}+k\left[\widetilde{\mathcal{A}}_{A^{\prime}, 1}\right]+\left[\partial_{n} \widetilde{\mathcal{A}}_{A^{\prime}, 2}\right]
$$

where $R_{A^{\prime}}: H^{s}(\Gamma) \rightarrow H^{s+1}(\Gamma)$ and $\widetilde{\mathcal{A}}_{A^{\prime}}$ satisfy for some constants $C, \gamma>0$ and a tubular neighborhood $T$ of $\Gamma$ that are all independent of $k \geq k_{0}$

$$
\begin{array}{ll}
\left\|R_{A^{\prime}}\right\|_{H^{s+1}(\Gamma) \leftarrow H^{s}(\Gamma)} \leq C k, & \left\|R_{A^{\prime}}\right\|_{H^{s}(\Gamma) \leftarrow H^{s}(\Gamma)} \leq q, \\
\left.\widetilde{\mathcal{A}}_{A^{\prime}, 1} \varphi \in \mathfrak{A}\left(C C_{\varphi}, \gamma, T\right)\right), & C_{\varphi}=k\|\varphi\|_{H^{-3 / 2}(\Gamma)}+k^{d / 2}\|\varphi\|_{H^{-1}(\Gamma)}, \\
\left.\widetilde{\mathcal{A}}_{A^{\prime}, 2} \varphi \in \mathfrak{A}\left(C \widetilde{C}_{\varphi}, \gamma, T\right)\right), & \widetilde{C}_{\varphi}=k\|\varphi\|_{H^{-3 / 2}(\Gamma)} .
\end{array}
$$

Proof. The proof is very similar to that of Lemma 5.7. We start from (5.2). Using the frequency splitting operators $H_{\Gamma}^{\text {neg }}$ and $L_{\Gamma}^{\text {neg }}$ of Lemma 5.3 , we can define

$$
\begin{aligned}
R_{A^{\prime}} & =H_{\Gamma}^{n e g}\left(\gamma_{1}^{i n t} \widetilde{S}_{V, p w}+\mathbf{i} \eta \gamma_{0}^{i n t} \widetilde{S}_{V, p w}+\mathbf{i} \eta V_{0}\right), \\
\widetilde{\mathcal{A}}_{A^{\prime}, 1} & =k^{-1} \chi_{\Omega}\left(-\mathbf{i} \eta \widetilde{\mathcal{A}}_{V, p w}-L_{\Gamma}^{n e g}\left(\gamma_{1}^{i n t} \widetilde{S}_{V, p w}+\mathbf{i} \eta \gamma_{0}^{i n t} \widetilde{S}_{V, p w}+\mathbf{i} \eta V_{0}\right)\right), \\
\widetilde{\mathcal{A}}_{A^{\prime}, 2} & =-\chi_{\Omega} \widetilde{\mathcal{A}}_{V, p w} .
\end{aligned}
$$

Using the mapping properties of $\widetilde{S}_{V, p w}$ and $V_{0}$ we can infer from Lemma 5.3 that $R_{A^{\prime}}$ has the desired mapping properties. For the operators $\widetilde{\mathcal{A}}_{A^{\prime}, 1}, \widetilde{\mathcal{A}}_{A^{\prime}, 2}$ we get from Theorem 4.3 and the mapping properties of $V_{0}$ that

$$
\left\|\gamma_{1}^{i n t} \widetilde{S}_{V, p w}+\mathbf{i} \eta \gamma_{0}^{i n t} \widetilde{S}_{V, p w}+\mathbf{i} \eta V_{0}\right\|_{L^{2}(\Gamma) \leftarrow H^{-1}(\Gamma)} \leq C k .
$$


From Lemma 5.3 we therefore get

$$
\widetilde{A}_{A^{\prime}, 1} \varphi \in \mathfrak{A}\left(C C_{\varphi}, \gamma, T\right), \quad C_{\varphi}=k^{d / 2}\|\varphi\|_{H^{-1}(\Gamma)}+k\|\varphi\|_{H^{-3 / 2}(\Gamma)}
$$

and an analogous estimate for $\widetilde{\mathcal{A}}_{A^{\prime}, 2}$.

REMARK 5.10. The proof of Lemma 5.9 shows that in the context of smooth domains, further decompositions are possible. In particular, it is possible to exploit the smoothing properties of $K_{0}$ and $K_{0}^{\prime}$. Since $K_{0}: L^{2}(\Gamma) \rightarrow H^{1}(\Gamma)$ and $K_{0}^{\prime}: L^{2}(\Gamma) \rightarrow H^{1}(\Gamma)$ we see that the splittings $K_{0}=H_{\Gamma}^{n e g} K_{0}+L_{\Gamma}^{n e g} K_{0}$ and $K_{0}^{\prime}=H_{\Gamma}^{n e g} K_{0}^{\prime}+L_{\Gamma}^{n e g} K_{0}^{\prime}$ lead, for example, to

$$
\left\|H_{\Gamma}^{n e g} K_{0}\right\|_{L^{2} \leftarrow L^{2}} \leq C q / k, \quad\left\|H_{\Gamma}^{n e g} K_{0}^{\prime}\right\|_{L^{2} \leftarrow L^{2}} \leq C q / k .
$$

Inserting this in the decompositions of Lemmata 5.5, 5.9 shows that the operators $A$, $A^{\prime}$ can be we written as sums of three terms: $\pm 1 / 2 \mathrm{Id}$, an operator that is small (as an operator $\left.L^{2}(\Gamma) \rightarrow L^{2}(\Gamma)\right)$, and an operator that maps into a trace class of analytic functions.

6. decomposition of the inverse of combined field operators. On smooth surfaces, it is well-known, [5,9], that the operators $A$ and $A^{\prime}$ of the form given in (1.3b), (1.4b) (i.e., the operator $1 / 2+K-\mathbf{i} \eta V$ and $1 / 2+K^{\prime}+\mathbf{i} \eta V$ ) are invertible as operators acting on $L^{2}(\Gamma)$. In fact, $1 / 2+K-\mathbf{i} \eta V$ is invertible as an operator on $H^{s}(\Gamma)$ for $s \geq 0$ and $1 / 2+K^{\prime}+\mathbf{i} \eta V$ as an operator on $H^{s}(\Gamma), s \geq-1 / 2,[6,7]$. Since the decomposition of their inverses is the primary aim of the paper, we state the main results of this section here:

TheOREM 6.1. Let $\Gamma$ be analytic and choose $s, s_{A} \geq 0$. Let $A=1 / 2+K-\mathbf{i} \eta V$. Then the decomposition of $A^{-1}$ given in Theorem 6.11 is valid.

Proof. Since the $L^{2}(\Gamma)$-invertibility of $A$ is known, Lemma 6.4 below shows that $A$ is invertible on $H^{s}(\Gamma)$ for arbitary $s \geq 0$. The result then follows from Theorem 6.11 . $\square$ TheOREM 6.2. Let $\Gamma$ be analytic and choose $s, s_{A} \geq-1 / 2$. Let $A^{\prime}=1 / 2+K^{\prime}+\mathbf{i} \eta V$. Then the decomposition of $\left(A^{\prime}\right)^{-1}$ given in Theorem 6.13 is valid.

Proof. Since the $H^{-1 / 2}(\Gamma)$-invertibility of $A^{\prime}$ is known, Lemma 6.4 shows that $A^{\prime}$ is invertible on $H^{s}(\Gamma)$ for arbitary $s \geq-1 / 2$. The statement then follows from Theorems $6.12,6.13$.

The decomposition results Theorem 6.1, 6.2 involve the norms $\left\|A^{-1}\right\|_{H^{s_{A}} \leftarrow H^{s_{A}}}$ and

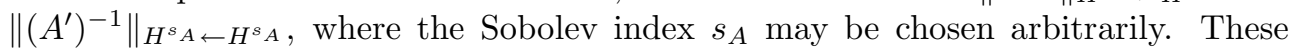
decomposition results are particularly useful if the $k$-dependence of these norms is available. In general, little is known about their $k$-dependence. A notable exception are star-shaped domains, for which the following was recently been shown:

LEMma 6.3 ( [7]). Let the Lipschitz domain $\Omega$ be star-shaped with respect to the origin. Then there exists a constant $C>0$ independent of $k$ such that for the operators $A_{k}$, $A_{k}^{\prime}$ given in (1.3b), (1.4b), there holds

$$
\left\|A_{k}^{-1}\right\|_{L^{2} \leftarrow L^{2}}=\left\|\left(A_{-k}^{\prime}\right)^{-1}\right\|_{L^{2} \leftarrow L^{2}} \leq C .
$$

Less is known about the invertibility properties of the operators (1.3a) and (1.4a). Nevertheless, due to the smoothness of $\Gamma$, we know that the invertibility of the operators $A$ and $A^{\prime}$ on one Sobolev space $H^{s}(\Gamma)$ implies invertibility on all spaces $H^{s^{\prime}}(\Gamma)$, $s^{\prime} \geq s:$

Lemma 6.4. Let $\Gamma$ be analytic. If $A$ is boundedly invertible on $H^{s}(\Gamma)$ for some $s \geq 0$, then it is boundedly invertible on $H^{s^{\prime}}(\Gamma)$ for all $s^{\prime} \geq s$. If $A^{\prime}$ is boundedly invertible on $H^{s}(\Gamma)$ for some $s \geq-1 / 2$, then it is boundedly invertible on $H^{s^{\prime}}(\Gamma)$ for all $s^{\prime} \geq s$. 
Proof. This follows from elliptic regularity and the jump conditions satisfied by the potential operators $\widetilde{V}$ and $\widetilde{K}$. We illustrate a typical case, namely, $A=-1 / 2+$ $K-\mathbf{i} \eta V$. Let $A$ be boundedly invertible on $H^{s}(\Gamma)$ for some $s \geq 0$ and let $s^{\prime}>s$. Let $\varphi \in H^{s}(\Gamma)$ solve $A \varphi=f \in H^{s^{\prime}}(\Gamma)$. Then $\varphi \in H^{\min \left\{1, s^{\prime}\right\}}(\Gamma)$, which follows from the smoothing properties $K_{0}: L^{2} \rightarrow H^{1}$ and $V_{0}: L^{2} \rightarrow H^{1}$. Hence, $A$ is boundedly invertible on $H^{s^{\prime}}(\Gamma)$ for $s \leq s^{\prime} \leq 1$. For $s^{\prime}>1$, we start by noting that we may assume that $A$ is boundedly invertible on $H^{\widetilde{s}}(\Gamma)$ for some $\widetilde{s} \geq 1 / 2$. Consider the potential $u=\widetilde{K} \varphi-\mathbf{i} \eta \widetilde{V} \varphi$, where $\varphi \in H^{\widetilde{s}}(\Gamma)$ solves $A \varphi=f \in H^{s^{\prime}}(\Gamma)$. Then $u$ satisfies in $\Omega$ the homogeneous Helmholtz equation and the boundary condition $\gamma_{0}^{i n t} u=(-1 / 2+K-\mathbf{i} \eta V) \varphi=f$. Elliptic regularity then ensures $u \in H^{s^{\prime}+1 / 2}(\Omega)$. Since $u$ satisfies the jump conditions

$$
[u]=\varphi, \quad\left[\partial_{n} u\right]=\mathbf{i} \eta \varphi
$$

we see that in $\Omega^{+}$the potential $u$ satisfies the homogeneous Helmholtz equation together with $\gamma_{1}^{\text {ext }} u-\mathbf{i} \eta \gamma_{0}^{\text {ext }} u=\gamma_{1}^{\text {int }} u-\mathbf{i} \eta \gamma_{0}^{\text {int }} u \in H^{s^{\prime}-1}(\Gamma)$. Elliptic regularity then gives $u \in H^{s^{\prime}+1 / 2}\left(\Omega_{R} \cap \Omega^{+}\right)$. The jump conditions (6.1) finally lead to $\varphi \in H^{s^{\prime}}(\Gamma)$.

6.1. analytic regularity. In this section, we study the equations $A \varphi=f$ and $A^{\prime} \varphi=f$ for analytic $\Gamma$ and analytic right-hand side $f$. The solution $\varphi$ is then likewise analytic and our goal is to study the $k$-dependence of the solution $\varphi$.

6.1.1. the operator $A$. LEMMA 6.5. Let $\Gamma$ be analytic and let $T$ be a tubular neighborhood of $\Gamma$. Suppose $g \in \mathfrak{A}\left(C_{g}, \gamma_{g}, T \backslash \Gamma\right)$ for some $C_{g}, \gamma_{g}>0$. Let $\varphi \in H^{1 / 2}(\Gamma)$ satisfy

$$
\left( \pm \frac{1}{2}+K-\mathbf{i} \eta V\right) \varphi=\gamma_{0}^{e x t} g-\gamma_{0}^{i n t} g
$$

Then $\varphi=\gamma_{0}^{e x t} u-\gamma_{0}^{i n t} u$, where, with the operator $\widetilde{A}$ defined in (6.2),

$$
u \in \mathfrak{A}\left(C C_{u}, \gamma, \Omega_{R}\right), \quad C_{u}=C_{g}+k^{-1}\|\nabla \widetilde{A} \varphi\|_{L^{2}\left(\Omega_{R}\right)}+\|\widetilde{A} \varphi\|_{L^{2}\left(\Omega_{R}\right)} .
$$

The constants $C$ and $\gamma$ depend solely on $\Gamma, \gamma_{g}, k_{0}$, and the choice of $R$.

Proof. Before proving the lemma, we stress the following points: First, the existence of $\varphi$ is stipulated as an assumption. Second, as will be discussed in more detail below, $k^{-1}\|\nabla \widetilde{A} \varphi\|_{L^{2}\left(\Omega_{R}\right)}+\|\widetilde{A} \varphi\|_{L^{2}\left(\Omega_{R}\right)}$ grows only algebraically in $k$ under appropriate assumptions. Thirdly, it is allowed to select $g$ such that it vanishes in $\Omega$ or in $\Omega^{+}$; in fact, this is how Lemma 6.5 will be employed below. Finally, in view of Lemma B.5 it is possible to select $u$ such that it vanishes on $\Omega$ or $\Omega^{+}$.

We define the potential $u$ on $\Omega \cup \Omega^{+}$by

$$
u=\widetilde{A} \varphi:=\widetilde{K} \varphi-\mathbf{i} \eta \widetilde{V} \varphi
$$

Then $u$ satisfies the homogeneous Helmholtz equation on $\Omega \cup \Omega^{+}$together with

$$
\begin{array}{ll}
\gamma_{0}^{i n t} u=[g] & \text { if }\left(-\frac{1}{2}+K-\mathbf{i} \eta V\right) \varphi=[g], \\
\gamma_{0}^{e x t} u=[g] & \text { if }\left(\frac{1}{2}+K-\mathbf{i} \eta V\right) \varphi=[g] .
\end{array}
$$

We will only consider the first case (corresponding to an interior Dirichlet problem) the method of proof can be applied to the second case as well. Also, for simplicity of notation we assume that $g=0$ on $\Omega^{+}$. This is not a restriction and can realized with the aid of Lemma B.5. 
The jump relations satisfied by $\widetilde{K}$ and $\widetilde{V}$ (see [18, Thm. 6.11]) give us on $\Gamma$ :

$$
[u]=\varphi, \quad \gamma_{1}^{e x t} u-\gamma_{1}^{i n t} u=\mathbf{i} \eta \varphi .
$$

The first jump relation shows that we have to prove $u \in \mathfrak{A}\left(C_{u}, \gamma_{u}, \Omega_{R}\right)$. To that end, we note that $u$ solves by (6.3)

$$
-\Delta u-k^{2} u=0 \quad \text { on } \Omega, \quad \gamma_{0}^{i n t} u=g .
$$

In view of the analyticity of $\Gamma$ and $g$, Theorem B.2 implies the existence of a tubular neighborhood $T$ of $\Gamma$ such that $u \in \mathfrak{A}\left(C_{1}, \gamma, T \cap \Omega\right)$, where $C_{1} \leq C\left(C_{g}+k^{-1}\|u\|_{\mathcal{H}, \Omega}\right)$ for a $C>0$ independent of $u$ and $k$.

The jump relations (6.5) imply the Robin boundary conditions

$$
\gamma_{1}^{e x t} u-\mathbf{i} \eta \gamma_{0}^{e x t} u=\gamma_{1}^{i n t} u-\mathbf{i} \eta \gamma_{0}^{i n t} u=: \widetilde{g}
$$

The analyticity of $\Gamma$ implies the existence of a tubular neighborhood of $\Gamma$ (again denoted $T)$ and an analytic function $G^{-} \in \mathfrak{A}\left(C C_{1} k, \gamma, T \cap \Omega\right)$ with $\gamma_{0}^{i n t} G^{-}=\widetilde{g}$. Next, Lemma B.5 implies the existence of a function $G$ and a tubular neighborhood of $\Gamma$ (again denoted $T$ ) with $G \in \mathfrak{A}\left(C C_{1} k, \gamma, T \cap \Omega^{+}\right)$and $\gamma_{0}^{e x t} G=\gamma_{0}^{\text {int }} G^{-}=\widetilde{g}$. Then, Theorem B.3 gives $u \in \mathfrak{A}\left(C C_{2}, \gamma, T \cap \Omega^{+}\right)$, where $C_{2}=C_{1}+k^{-1}\|u\|_{\mathcal{H}, \Omega^{+} \cap B_{R}}$. Since $u=\widetilde{A} \varphi$, we have so far obtained $u \in \mathfrak{A}\left(C C_{u}, \gamma, T \backslash \Gamma\right)$ with $C_{u}$ defined in the statement of the lemma. Interior regularity (see [19, Prop. 5.5.1]) finally gives estimates for $u$ not only near $\Gamma$ but in all of $\Omega_{R}$, i.e., $u \in \mathfrak{A}\left(C C_{u}, \gamma_{u}, \Omega_{R}\right)$ for suitable $C, \gamma_{u}>0$. $\square$ The existence of $\varphi$ is stipulated as an assumption in Lemma 6.5. We formulated $\varphi \in H^{1 / 2}(\Gamma)$ since this readily implies $\widetilde{A} \varphi \in H^{1}\left(\Omega_{R}\right)$ and the constant $C_{u}$ can be estimated in terms of $\|\varphi\|_{H^{1 / 2}(\Gamma)}$. However, it will be more convenient in the following to bound $C_{u}$ in terms of $\|\varphi\|_{L^{2}(\Gamma)}$ and $\|A \varphi\|_{H^{1 / 2}(\Gamma)}$, which we now show how to do:

Lemma 6.6. Assume the hypotheses of Lemma 6.5. If $\varphi \in H^{1 / 2}(\Gamma)$ then

$\|\widetilde{A} \varphi\|_{L^{2}\left(\Omega_{R}\right)}+k^{-1}\|\nabla \widetilde{A} \varphi\|_{L^{2}\left(\Omega_{R}\right)} \leq C\left[k^{-1}\|\varphi\|_{H^{1 / 2}(\Gamma)}+k\|\varphi\|_{H^{-1 / 2}(\Gamma)}+k^{2}\|\varphi\|_{H^{-3 / 2}(\Gamma)}\right]$.

If $\varphi \in L^{2}(\Gamma)$ and $A \varphi \in H^{1 / 2}(\Gamma)$ then

$\|\widetilde{A} \varphi\|_{L^{2}\left(\Omega_{R}\right)}+k^{-1}\|\nabla \widetilde{A} \varphi\|_{L^{2}\left(\Omega_{R}\right)} \leq C\left[\|A \varphi\|_{H^{1 / 2}(\Gamma)}+k^{2}\|\varphi\|_{H^{-1 / 2}(\Gamma)}+k^{3}\|\varphi\|_{H^{-3 / 2}(\Gamma)}\right]$.

Proof. If $\varphi \in H^{1 / 2}(\Gamma)$, then we can insert the result of Corollary 4.5 to get

$$
\begin{aligned}
\|\widetilde{A} \varphi\|_{L^{2}\left(\Omega_{R}\right)} & \leq C\left[k\|\varphi\|_{H^{-1 / 2}(\Gamma)}+k^{2}\|\varphi\|_{H^{-3 / 2}(\Gamma)}\right], \\
\|\nabla \widetilde{A} \varphi\|_{L^{2}\left(\Omega_{R}\right)} & \leq C\left[\|\varphi\|_{H^{1 / 2}(\Gamma)}+k^{2}\|\varphi\|_{H^{-1 / 2}(\Gamma)}+k^{3}\|\varphi\|_{H^{-3 / 2}(\Gamma)}\right],
\end{aligned}
$$

which is the first estimate. For the second one, we consider again the case where $\widetilde{A} \varphi$ (see (6.2)) solves an interior Dirichlet problem. If $\varphi \in L^{2}(\Gamma)$, then it is a priori not clear that $\widetilde{A} \varphi \in H^{1}\left(\Omega_{R}\right)$. However, this can be inferred as follows: We write $A= \pm 1 / 2+K_{0}+S$, where, by Theorem 4.4, the operator $S: L^{2}(\Gamma) \rightarrow H^{1}(\Gamma)$. Since likewise $K_{0}: L^{2}(\Gamma) \rightarrow H^{1}(\Gamma)$, we conclude from $A \varphi \in H^{1 / 2}(\Gamma)$ that $1 / 2 \varphi \in H^{1 / 2}(\Gamma)$. In particular, $\widetilde{A} \varphi \in H^{1}\left(\Omega_{R}\right)$. To get bounds for $u:=\widetilde{A} \varphi$, we restrict our attention to the case $A=-1 / 2+K-\mathbf{i} \eta V$ as in the proof of Lemma 6.5 and note that (4.21)-(4.23) of Corollary 4.5 produce

$$
k^{2}\|u\|_{H^{-1}(\Omega)}+k^{2}\|u\|_{H^{-1}\left(B_{2 R}\right)} \leq \underset{21}{C\left[k^{2}\|\varphi\|_{H^{-1 / 2}(\Gamma)}+k^{3}\|\varphi\|_{H^{-3 / 2}(\Gamma)}\right] .}
$$


Next, $u$ is the solution of the following interior Dirichlet problem:

$$
-\Delta u=k^{2} \widetilde{A} \varphi \in L^{2}(\Omega) \quad \text { in } \Omega, \quad \gamma_{0}^{i n t} u=g:=A \varphi .
$$

Standard a priori bounds for Laplace Dirichlet problems together with (6.7) and (6.7) imply

$$
\|u\|_{\mathcal{H}, \Omega} \leq C\left[\|g\|_{H^{1 / 2}(\Gamma)}+k^{2}\|\varphi\|_{H^{-1 / 2}(\Gamma)}+k^{3}\|\varphi\|_{H^{-3 / 2}(\Gamma)}\right] .
$$

Lemma 4.6 allows us to infer

$$
\left\|\gamma_{1}^{i n t} u\right\|_{H^{-1 / 2}(\Gamma)} \leq C k\|u\|_{\mathcal{H}, \Omega}, \quad\|u\|_{H^{1 / 2}\left(\partial B_{R}\right)} \leq C k^{2}\|u\|_{H^{-1}\left(B_{2 R}\right)} .
$$

The jump condition (6.5) satisfied by $u$ reads $\gamma_{1}^{e x t} u-\gamma_{1}^{\text {int } u} u=\mathbf{i} \eta \varphi$. Rewriting this as $\gamma_{1}^{\text {ext }} u=\gamma_{1}^{\text {int }} u+\mathbf{i} k \varphi$, we infer that $u$ solves in $\Omega^{+}$

$$
-\Delta u=k^{2} u \quad \text { on } \Omega^{+}, \quad \gamma_{1}^{e x t} u=\gamma_{1}^{i n t} u+\mathbf{i} k \varphi,\left.\quad u\right|_{\partial B_{R}}=\left.u\right|_{\partial B_{R}} .
$$

A priori bounds for the Laplace operator together with (6.8) give us

$$
\begin{aligned}
\|u\|_{H^{1}\left(\Omega_{R} \backslash \bar{\Omega}\right)} & \leq C\left[\left\|k^{2} u\right\|_{H^{-1}\left(\Omega_{R} \backslash \bar{\Omega}\right)}+\left\|\gamma_{1}^{e x t} u\right\|_{H^{-1 / 2}(\Gamma)}+k\|\varphi\|_{H^{-1 / 2}(\Gamma)}+\|u\|_{H^{1 / 2}\left(\partial B_{R}\right)}\right] \\
& \leq C k\left[\|g\|_{H^{1 / 2}(\Gamma)}+k^{2}\|\varphi\|_{H^{-1 / 2}(\Gamma)}+k^{3}\|\varphi\|_{H^{-3 / 2}(\Gamma)}\right]
\end{aligned}
$$

which concludes the argument. $\square$

If the operator $A$ is invertible on $H^{s_{A}}(\Gamma)$ and a bound on $\left\|A^{-1}\right\|_{H^{s_{A} \leftarrow H^{s_{A}}}}$ is available, then then we obtain the following regularity assertion for $A^{-1}$ :

COROLlary 6.7. Let $\Gamma$ be analytic, $T$ be a tubular neighborhood of $\Gamma$, and $C_{g}, \gamma_{g}>0$. Let $A$ be boundedly invertible on $H^{s_{A}}(\Gamma)$ for some $s_{A} \geq 0$. Then there exist constants $C, \gamma>0$ such that for every $g \in \mathfrak{A}\left(C_{g}, \gamma_{g}, T \backslash \Gamma\right)$ the solution $\varphi \in H^{s_{A}}(\Gamma)$ of $A \varphi=[g]$ satisfies

$\varphi=[u], \quad u \in \mathfrak{A}\left(C C_{\varphi}, \gamma, \Omega_{R}\right), \quad C_{\varphi}:=C_{g} k\left(1+k^{\beta}\left\|A^{-1}\right\|_{H^{s_{A} \leftarrow H^{s_{A}}}}\right) \quad \beta:=\frac{5}{2}+s_{A}$.

Furthermore, $u$ is given explicitly by (6.2), i.e., $u=\widetilde{A}\left(A^{-1}[g]\right)$.

Proof. From the trace inequality (and, in the limiting case $s_{A}=0$, a multiplicative trace inequality) we get

$$
\|[g]\|_{H^{s_{A}(\Gamma)}} \leq C C_{g} k^{s_{A}+1 / 2}, \quad\|[g]\|_{L^{2}(\Gamma)} \leq C C_{g} k^{1 / 2}, \quad\|[g]\|_{H^{1 / 2}(\Gamma)} \leq C C_{g} k .
$$

Therefore, by assumption we obtain for $\varphi=A^{-1}[g]$

$$
\|\varphi\|_{L^{2}(\Gamma)} \leq C\|\varphi\|_{H^{s_{A}(\Gamma)}} \leq C C\left(A, s_{A}, k\right)\|[g]\|_{H^{s_{A}(\Gamma)}} \leq C\left\|A^{-1}\right\|_{H^{s_{A} \leftarrow H^{s_{A}}}} k^{s_{A}+1 / 2} C_{g} .
$$

Lemma 6.6 then implies for the function $u=\widetilde{A} \varphi$

$$
\|u\|_{L^{2}\left(\Omega_{R}\right)}+k^{-1}\|\nabla u\|_{L^{2}\left(\Omega_{R}\right)} \leq C k^{7 / 2+s_{A}} C_{g}\left\|A^{-1}\right\|_{H^{s_{A} \leftarrow H^{s_{A}}} .} .
$$

An appeal to Lemma 6.5 concludes the argument. 
6.1.2. the operator $A^{\prime}$. For the operator $A^{\prime}$, one can proceed very similarly as for the operator $A$.

LEMMA 6.8. Let $T$ be a tubular neighborhood of $\Gamma$ and let $g_{1} \in \mathfrak{A}\left(C_{g_{1}}, \gamma_{1}, T \backslash \Gamma\right)$ and $g_{2} \in \mathfrak{A}\left(C_{g_{2}}, \gamma_{2}, T \backslash \Gamma\right)$. Let $\varphi \in H^{-1 / 2}(\Gamma)$ satisfy

$$
\left( \pm \frac{1}{2}+K^{\prime}+\mathbf{i} \eta V\right) \varphi=k\left(\gamma_{0}^{e x t} g_{1}-\gamma_{0}^{i n t} g_{1}\right)+\left(\gamma_{1}^{e x t} g_{2}-\gamma_{1}^{i n t} g_{2}\right)
$$

Then $\varphi=\gamma_{1}^{\text {ext }} u-\gamma_{1}^{\text {int }} u$ for a function

$$
u \in \mathfrak{A}\left(C C_{u}, \Omega_{R}\right) \quad C_{u}=C_{g_{1}}+C_{g_{2}}+k^{-1}\|\varphi\|_{H^{-1 / 2}(\Gamma)}+k\|\varphi\|_{H^{-3 / 2}(\Gamma)} .
$$

The constants $C, \gamma>0$ depend only on $\Gamma, \gamma_{g_{1}}, \gamma_{g_{2}}$, and $k_{0}$.

Proof. We introduce the potential $u:=\widetilde{V} \varphi$, which satisfies the homogeneous Helmholtz equation in $\Omega \cup \Omega^{+}$. Additionally, it satisfies the jump conditions $\gamma_{0}^{\text {int }} u=\gamma_{0}^{\text {ext }} u$ and

$\gamma_{1}^{i n t} u+\mathbf{i} \eta u=\left(\frac{1}{2}+K^{\prime}+\mathbf{i} \eta V\right) \varphi \quad$ and $\quad \gamma_{1}^{e x t} u+\mathbf{i} \eta u=\left(-\frac{1}{2}+K^{\prime}+\mathbf{i} \eta V\right) \varphi \quad$ on $\Gamma$.

Let us assume that $A^{\prime}=1 / 2+K^{\prime}+\mathbf{i} \eta V$, since the case of $A^{\prime}=-1 / 2+K^{\prime}+\mathbf{i} \eta V$ is handled with analogous arguments. For simplicity of notation, we assume, as we may in view of Lemma B.5, that $g_{1}=g_{2}=0$ on $\Omega^{+}$.

Then $u$ solves the homogeneous Helmholtz equation in $\Omega$ with Robin boundary condition $\gamma_{1}^{i n t} u+\mathbf{i} \eta u=k \gamma_{0}^{i n t} g_{1}+\gamma_{1}^{i n t} g_{2}$ on $\Gamma$. The analyticity of $g_{1}$ and $g_{2}$ then implies by Theorem B.3 the existence of a tubular neighborhood $T^{\prime}$ of $\Gamma$ and a constant $\gamma>0$ such that

$$
u \in \mathfrak{A}\left(C C_{u}^{\prime}, \gamma, T^{\prime} \cap \Omega\right), \quad C_{u}^{\prime}:=\left[k^{-1}\|\nabla u\|_{L^{2}(\Omega)}+\|u\|_{L^{2}(\Omega)}+C_{g_{1}}+C_{g_{2}}\right]
$$

By means of Lemma B.5, we may view $\gamma_{0}^{\text {int } u}$ as the trace $\gamma_{0}^{\text {ext }} \widetilde{u}$ of a function $\widetilde{u} \in$ $\mathfrak{A}\left(C C_{u}^{\prime}, \widetilde{\gamma}, T^{\prime \prime} \cap \Omega^{+}\right)$, where the tubular neighborhood $T^{\prime \prime}$ and the constant $\widetilde{\gamma}$ depend solely on $\Gamma, \gamma$, and $k_{0}$. In $\Omega^{+}$, the function $u$ satisfies the homogeneous Helmholtz equation and, in view of the jump condition $\gamma_{0}^{\text {ext }} u=\gamma_{0}^{i n t} u$, on $\Gamma$ the Dirichlet boundary condition $\gamma_{0}^{e x t} u=\gamma_{0}^{e x t} \widetilde{u}$. Hence, we conclude from Theorem B.2 the existence of a tubular neighborhood (again denoted $T$ ) and constants $C, \gamma_{u}>0$ that depend solely on $\Gamma$ and $\widetilde{\gamma}$ such that

$$
u \in \mathfrak{A}\left(C C_{u}^{\prime \prime}, \gamma_{u}, T \cap \Omega^{+}\right), \quad C_{u}^{\prime \prime}=C_{u}^{\prime}+k^{-1}\|u\|_{\mathcal{H}, \Omega^{+} \cap B_{R}} .
$$

Corollary 4.5 implies

$$
k^{-1}\|u\|_{\mathcal{H}, B_{2 R}} \leq C\left[k^{-1}\|\varphi\|_{H^{-1 / 2}(\Gamma)}+k\|\varphi\|_{H^{-3 / 2}(\Gamma)}\right]
$$

so that we conclude $u \in \mathfrak{A}\left(C C_{u}, \gamma_{u}, T \backslash \Gamma\right)$ with $C_{u}$ defined in the statement of the lemma. Finally, interior regularity (see [19, Prop. 5.5.1]) gives estimates not only near $\Gamma$ but in all of $\Omega_{R}$, i.e., $u \in \mathfrak{A}\left(C C_{\varphi}, \gamma_{u}, \Omega_{R}\right)$ for suitable $\gamma_{u}, C>0$. Observing that $\gamma_{1}^{\text {int }} u-\gamma_{1}^{\text {ext }} u=\varphi$ concludes the proof. $\square$

Corollary 6.9. Let $\Gamma$ be analytic, $T$ be a tubular neighborhood of $\Gamma$, and $C_{g_{1}}, C_{g_{2}}$, $\gamma_{g}>0$. Let $A^{\prime}$ be boundedly invertible on $H^{s_{A}}(\Gamma)$ for some $s_{A} \geq-1 / 2$. Then there exist constants $C, \gamma>0$ independent of $k \geq k_{0}$ such that for all $g_{1} \in \mathfrak{A}\left(C_{g_{1}}, \gamma_{g}, T \backslash \Gamma\right)$, $g_{2} \in \mathfrak{A}\left(C_{g_{2}}, \gamma_{g}, T \backslash \Gamma\right)$ the solution $\varphi \in H^{s_{A}}(\Gamma)$ of $A \varphi=k\left[g_{1}\right]+\left[\partial_{n} g_{2}\right]$ satisfies

$$
\begin{aligned}
& \varphi=\left[\partial_{n} u\right], \quad u \in \mathfrak{A}\left(C C_{\varphi}, \gamma, \Omega_{R}\right), \quad C_{\varphi}:=\left(C_{g_{1}}+C_{g_{2}}\right)\left(1+k^{\beta}\left\|\left(A^{\prime}\right)^{-1}\right\|_{H^{s} A \leftarrow H^{s} A}\right), \\
& \beta=\frac{5}{2}+s_{A}^{+}, \quad s_{A}^{+}:=\max \left\{s_{A}, 0\right\} .
\end{aligned}
$$


Furthermore, $u$ is given explicitly as $u=\widetilde{V}\left(\left(A^{\prime}\right)^{-1}[g]\right)$.

Proof. We use Lemma 6.8. Using $s_{A} \geq-1 / 2$ and the assumption that $A^{\prime}$ is invertible on $H^{s_{A}}(\Gamma)$ gives for the solution $\varphi$ of $A^{\prime} \varphi=k\left[g_{1}\right]+\left[\partial_{n} g_{2}\right]$

$$
\begin{aligned}
& \|\varphi\|_{H^{-1 / 2}(\Gamma)} \leq C\|\varphi\|_{H^{s_{A}(\Gamma)}} \leq C\left\|\left(A^{\prime}\right)^{-1}\right\|_{H^{s_{A} \leftarrow H^{s_{A}}}}\left\|k\left[g_{1}\right]+\left[\partial_{n} g_{2}\right]\right\|_{H^{s_{A}}(\Gamma)}
\end{aligned}
$$

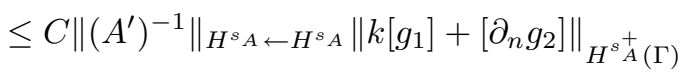

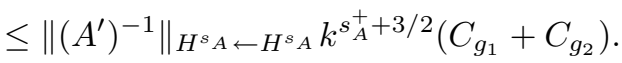

Hence, we get

$C_{g_{1}}+C_{g_{2}}+k^{-1}\|\varphi\|_{H^{-1 / 2}(\Gamma)}+k\|\varphi\|_{H^{-3 / 2}(\Gamma)} \leq C\left(C_{g_{1}}+C_{g_{2}}\right)\left(1+k^{5 / 2+s_{A}^{+}}\left\|\left(A^{\prime}\right)^{-1}\right\|_{H^{s_{A} \leftarrow H^{s} A}}\right)$.

An appeal to Lemma 6.8 concludes the proof.

6.2. finite regularity. This section is the core of the paper and provides decomposition results for the operators $A^{-1}$ and $\left(A^{\prime}\right)^{-1}$ as operators acting on Sobolev spaces $H^{s}(\Gamma)$. These results are formulated as Theorems 6.11, 6.12. Before working out the details, we formulate a lemma that isolates an important structural element of the proof of Theorems 6.11, 6.12.

Lemma 6.10 ("iteration lemma"). Let $T$ be a tubular neighborhood of $\Gamma$. Let $s$, $s_{B} \in \mathbb{R}$, and $\gamma_{1}, \gamma_{2}, \gamma>0$ be given. Let $C_{\text {smooth }}(k), C_{\text {solve }}(k) \geq 0$ be two, possibly $k$-dependent numbers.

Assume that $B: H^{s}(\Gamma) \rightarrow H^{s}(\Gamma)$ satisfies the following conditions:

(i) $B$ can be decomposed as

$$
B=B_{0}+B_{\mathcal{A}}+R
$$

where $B_{0}: H^{s}(\Gamma) \rightarrow H^{s}(\Gamma)$ is boundedly invertible, $R$ is a bounded linear operator with

$$
\left\|R B_{0}^{-1}\right\|_{H^{s}(\Gamma) \leftarrow H^{s}(\Gamma)} \leq q<1
$$

and $B_{\mathcal{A}}$ is a bounded linear operator of the form

$$
B_{\mathcal{A}}=k\left[\widetilde{B}_{\mathcal{A}, 1}\right]+\left[\partial_{n} \widetilde{B}_{\mathcal{A}, 2}\right]
$$

with

$$
\widetilde{B}_{\mathcal{A}, i} \varphi \in \mathfrak{A}\left(C_{\text {smooth }}(k)\|\varphi\|_{H^{s}(\Gamma)}, \gamma, T \backslash \Gamma\right) \quad \forall \varphi \in H^{s}(\Gamma), \quad i \in\{1,2\} .
$$

(ii) $B^{-1}$ is a bounded linear operator in $H^{s}(\Gamma)$ and $H^{s_{B}}(\Gamma)$ (with possibly $k$-dependent norms).

(iii) If $\varphi \in H^{s}(\Gamma)$ satisfies $B \varphi=k\left[g_{1}\right]+\left[\partial_{n} g_{2}\right]$ for some $g_{1} \in \mathfrak{A}\left(C_{g_{1}}, \gamma_{1}, T \backslash \Gamma\right), g_{2} \in$ $\mathfrak{A}\left(C_{g_{2}}, \gamma_{2}, T \backslash \Gamma\right)$, then $\varphi=[u]\left(\right.$ or, $\left.\varphi=\left[\partial_{n} u\right]\right)$ for some $u \in \mathfrak{A}\left(C_{\text {solve }}(k)\left(C_{G_{1}}+\right.\right.$ $\left.\left.C_{G_{2}}\right), \gamma, \Omega_{R}\right)$.

Under these assumptions there exist constants $\widetilde{C}, \widetilde{\gamma}>0$ depending only on $\gamma_{1}, \gamma_{2}$, and $\Gamma$, and $k_{0}$ such that $B^{-1}$ can be written as

$$
B^{-1}=B_{Z}+B_{B}
$$

where $B_{B}$ has the form $B_{B} f=\left[\widetilde{B}_{B} f\right]$ (or $B_{B} f=\left[\partial_{n} \widetilde{B}_{B} f\right]$ ) and

$$
\begin{aligned}
& \left\|B_{Z}\right\|_{H^{s}(\Gamma) \leftarrow H^{s}(\Gamma)} \leq(1-q)^{-1}\left\|B_{0}^{-1}\right\|_{H^{s}(\Gamma) \leftarrow H^{s}(\Gamma)}, \\
& \widetilde{B}_{B} f \in \mathfrak{A}\left(C_{B}, \widetilde{\gamma}, \Omega_{R}\right), \quad C_{B}:=\widetilde{C} C_{\text {solve }}(k) C_{\text {smooth }}(k)\|f\|_{H^{s}(\Gamma)} .
\end{aligned}
$$


Proof. For $f \in H^{s}(\Gamma)$ consider the following iteration:

$$
\begin{aligned}
\varphi_{0}^{\text {finite }} & :=0, & & \varphi_{0}^{\mathcal{A}}:=0, \\
B_{0} \delta_{n}^{\text {finite }} & :=f-B\left(\varphi_{n}^{\text {finite }}+\varphi_{n}^{\mathcal{A}}\right), & & \\
B \delta_{n}^{\mathcal{A}} & :=-B_{\mathcal{A}} \delta_{n}^{\text {finite }}, & & \\
\varphi_{n+1}^{\text {finite }} & :=\varphi_{n}^{\text {finite }}+\delta_{n}^{\text {finite }}, & & \varphi_{n+1}^{\mathcal{A}}:=\varphi_{n}^{\mathcal{A}}+\delta_{n}^{\mathcal{A}} .
\end{aligned}
$$

The sequences $\left(\varphi_{n}^{\text {finite }}\right)_{n=0}^{\infty}$ and $\left(\varphi_{n}^{\mathcal{A}}\right)_{n=0}^{\infty}$ converge as we now show. Define the residual $r_{n}:=f-B\left(\varphi_{n}^{\text {finite }}+\varphi_{n}^{\mathcal{A}}\right)$. Then

$$
\begin{aligned}
r_{n+1} & =f-B\left(\varphi_{n+1}^{\text {finite }}+\varphi_{n+1}^{\mathcal{A}}\right)=f-B\left(\varphi_{n}^{\text {finite }}+\delta_{n}^{\text {finite }}+\varphi_{n}^{\mathcal{A}}+\delta_{n}^{\mathcal{A}}\right) \\
& =r_{n}-B \delta_{n}^{\text {finite }}-B \delta_{n}^{\mathcal{A}}=r_{n}-\left(B_{0}+B_{\mathcal{A}}+R\right) \delta_{n}^{\text {finite }}-B \delta_{n}^{\mathcal{A}} \\
& =-R \delta_{n}^{\text {finite }}-B_{\mathcal{A}} \delta_{n}^{\text {finite }}-B \delta_{n}^{\mathcal{A}}=-R \delta_{n}^{\text {finite }}=-R B_{0}^{-1} r_{n} .
\end{aligned}
$$

The assumption $\left\|R B_{0}^{-1}\right\|_{H^{s}(\Gamma) \leftarrow H^{s}(\Gamma)} \leq q<1$ therefore implies $\left\|r_{n}\right\|_{H^{s}(\Gamma)} \leq q^{n}\left\|r_{0}\right\|_{H^{s}(\Gamma)}$ and thus $\left\|\delta_{n}^{\text {finite }}\right\|_{H^{s}(\Gamma)} \leq q^{n}\left\|B_{0}^{-1}\right\|_{H^{s}(\Gamma) \leftarrow H^{s}(\Gamma)}\left\|r_{0}\right\|_{H^{s}(\Gamma)}$. We conclude that the sum $\sum_{n=0}^{\infty} \delta_{n}^{\text {finite }}$ converges in $H^{s}(\Gamma)$. Since $B$ is a bounded linear operator, also the sum $\sum_{n=0}^{\infty} \delta_{n}^{\mathcal{A}}$ converges in $H^{s}(\Gamma)$. We thus define the operators $B_{Z}$ and $B_{B}$ by

$$
B_{Z}: f \mapsto \lim _{n \rightarrow \infty} \varphi_{n}^{\text {finite }}=\sum_{n=0}^{\infty} \delta_{n}^{\text {finite }}, \quad B_{B}: f \mapsto \lim _{n \rightarrow \infty} \varphi_{n}^{\mathcal{A}}=\sum_{n=0}^{\infty} \delta_{n}^{\mathcal{A}} .
$$

It is easy to see that $\left\|B_{Z}\right\|_{H^{s}(\Gamma) \leftarrow H^{s}(\Gamma)} \leq(1-q)^{-1}\left\|B_{0}^{-1}\right\|_{H^{s}(\Gamma) \leftarrow H^{s}(\Gamma)}$. Next, in view of $\lim _{n \rightarrow \infty} \delta_{n}^{\text {finite }}=0$, we obtain from (6.11) that $\lim _{n \rightarrow \infty} \varphi_{n}^{\text {finite }}+\varphi_{n}^{\mathcal{A}}$ is the solution of $B \varphi=f$. To obtain the representation $B_{B} f=\left[\widetilde{B}_{B} f\right]\left(\right.$ or $\left.B_{B} f=\left[\partial_{n} \widetilde{B}_{B}\right]\right)$, we sum the terms in (6.12) to get the relation

$$
B\left(B_{B} f\right)=-B_{\mathcal{A}} B_{Z} f .
$$

Thus, by assumptions on the operators $B$ and $B_{\mathcal{A}}$, we see that $B_{B} f$ has the form $B_{B} f=\left[\widetilde{B}_{B} f\right]\left(\right.$ or $\left.\left[\partial_{n} \widetilde{B}_{B} f\right]\right)$ for an operator $\widetilde{B}_{B}$ that satisfies

$$
\widetilde{B}_{B} f \in \mathfrak{A}\left(C C_{\text {solve }}(k) C_{\text {smooth }}(k)\|f\|_{H^{s}(\Gamma)}, \widetilde{\gamma}, \Omega_{R}\right) .
$$

for appropriate $\widetilde{\gamma}$.

6.2.1. the operator $A$. We show that the operator $A^{-1}$ of (1.3) can be decomposed into a zero-th order operator with $k$-independent bounds and an analytic part.

Theorem 6.11 (decomposition of $A^{-1}$ ). Let $\Gamma$ be analytic, $s, s_{A} \geq 0$. Let $A$ be invertible on $H^{\min \left\{s, s_{A}\right\}}(\Gamma)$. Then there exist constants $C, \gamma>0$ independent of $k \geq k_{0}$ with the following properties: The operator $A^{-1}$ can be written as

$$
A^{-1}=A_{Z}+\gamma_{0}^{e x t} \widetilde{A}_{A^{-1}}-\gamma_{0}^{i n t} \widetilde{A}_{A^{-1}}
$$

where the linear operators $A_{Z}$ und $\widetilde{\mathcal{A}}_{A^{-1}}$ satisfy

$$
\begin{aligned}
& \left\|A_{Z}\right\|_{H^{s}(\Gamma) \leftarrow H^{s}(\Gamma)} \leq C, \\
& \widetilde{\mathcal{A}}_{A^{-1}} f \in \mathfrak{A}\left(C C_{f}, \gamma, \Omega_{R}\right), \quad C_{f}:=k^{3}\left(1+k^{5 / 2+s_{A}}\left\|A^{-1}\right\|_{H^{s_{A} \leftarrow H^{s_{A}}}}\right)\|f\|_{H^{s}(\Gamma)} .
\end{aligned}
$$


Proof. Before turning to the proof, we point out that, since only the jump of $\widetilde{A}_{A} f$ across $\Gamma$ appears in the decomposition of $A^{-1}$, there is some freedom in the choice of $\widetilde{A}_{A}$. In particular, $\widetilde{\mathcal{A}}_{A}$ can be selected such that $\left.\left(\widetilde{\mathcal{A}}_{A} f\right)\right|_{\Omega^{+}}=0$ or $\left.\left(\widetilde{\mathcal{A}}_{A} f\right)\right|_{\Omega}=0$. In fact, the proof shows that we construct $\widetilde{\mathcal{A}}_{A}$ such that $\widetilde{\mathcal{A}}_{A} f=0$ on $\Omega^{+}$if $A=$ $-1 / 2+K-\mathbf{i} \eta V$ is considered and $\widetilde{\mathcal{A}}_{A} f=0$ on $\Omega$ if $A=1 / 2+K-\mathbf{i} \eta V$. We also point out that, although $A$ is assumed to be invertible on $H^{s}(\Gamma)$, the bound

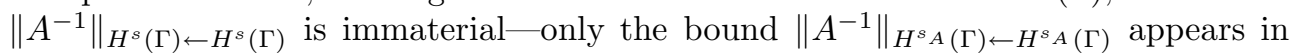
the estimates.

Our starting point is Lemma 2.5 , which asserts that $-1 / 2+K_{0}$ and $1 / 2+K_{0}+\mathbf{i} V_{0}$ are invertible operators on $H^{s}(\Gamma)$. Lemma 5.5 and Remark 5.6 permit us to write

$$
\begin{array}{ll}
A=-\frac{1}{2}+K_{0}+R_{A}+k\left[\widetilde{\mathcal{A}}_{A}\right], & \text { if } A=-1 / 2+K-\mathbf{i} \eta V, \\
A=\frac{1}{2}+K_{0}+\mathbf{i} V_{0}+R_{A}+k\left[\widetilde{\mathcal{A}}_{A}\right], & \text { if } A=1 / 2+K-\mathbf{i} \eta V .
\end{array}
$$

with operators $R_{A}$ and $\widetilde{\mathcal{A}}_{A}$ having the properties stated in Lemma 5.5. In the notation of Lemma 6.10, we set

$R=R_{A}, \quad \widetilde{B}_{\mathcal{A}, 1}=\widetilde{\mathcal{A}}_{A}, \quad \widetilde{B}_{\mathcal{A}, 2}=0, \quad B_{0}= \begin{cases}\frac{1}{2}+K_{0}+\mathbf{i} V_{0}, & \text { if } A=1 / 2+K-\mathbf{i} \eta V, \\ -\frac{1}{2}+K_{0} & \text { if } A=-1 / 2+K-\mathbf{i} \eta V\end{cases}$

In view of Lemma 5.5, the norm $\left\|R_{A}\right\|_{H^{s}(\Gamma) \leftarrow H^{s}(\Gamma)}$ can be made arbitrarily small. We may therefore assume that $\left\|R B_{0}^{-1}\right\|_{H^{s}(\Gamma) \leftarrow H^{s}(\Gamma)}<1$. Furthermore, Lemma 5.5 together with the trivial embedding $H^{s}(\Gamma) \subset H^{-1 / 2}(\Gamma) \subset H^{-3 / 2}(\Gamma)$ implies that $C_{\text {smooth }}(k) \leq C k$. Finally, Corollary 6.7 provides us, again in the terminology of Lemma 6.10, with

$$
C_{\text {solve }}(k) \sim k^{2}\left(1+k^{5 / 2+s_{A}} C\left(A, s_{A}, k\right)\right) .
$$

Thus, $C_{\text {solve }}(k) C_{\text {smooth }}(k) \sim k^{3}\left(1+k^{5 / 2+s_{A}} C\left(A, s_{A}, k\right)\right)$, and Lemma 6.10 implies the result.

6.2.2. the operator $A^{\prime}$. The operator $A^{\prime}$ is handled with similar techniques.

THEOREM 6.12 (decomposition of $\left(A^{\prime}\right)^{-1}$ ). Let $\Gamma$ be analytic, $s \geq 0, s_{A} \geq-1 / 2$. Let $A^{\prime}$ be boundedly invertible on $H^{\min \left\{s, s_{A}\right\}}(\Gamma)$. Then there exist constants $C, \gamma>0$ independent of $k \geq k_{0}$ with the following properties: The operator $\left(A^{\prime}\right)^{-1}$ can be written as

$$
\left(A^{\prime}\right)^{-1}=A_{Z}^{\prime}+\gamma_{1}^{e x t} \widetilde{A}_{A^{\prime}, i n v}-\gamma_{1}^{i n t} \widetilde{A}_{A^{\prime}, \text { inv }}
$$

where the linear operators $A_{A}^{\prime}$ and $\widetilde{A}_{A^{\prime}, \text { inv }}$ satisfy with $s_{A}^{+}:=\max \left\{s_{A}, 0\right\}$

$$
\begin{aligned}
& \left\|A_{Z}^{\prime}\right\|_{H^{s}(\Gamma) \leftarrow H^{s}(\Gamma)} \leq C, \\
& \widetilde{A}_{A^{\prime}, i n v} f \in \mathfrak{A}\left(C C_{f}, \gamma, \Omega_{R}\right), \quad C_{f}:=\left(1+k^{5 / 2+s_{A}^{+}}\left\|\left(A^{\prime}\right)^{-1}\right\|_{H^{s_{A} \leftarrow H^{s_{A}}}}\right)\|f\|_{H^{s}(\Gamma)} .
\end{aligned}
$$

Proof. We point out that, although $A^{\prime}$ is assumed to be invertible on $H^{s}(\Gamma)$, the bound $\left\|\left(A^{\prime}\right)^{-1}\right\|_{H^{s}(\Gamma) \leftarrow H^{s}(\Gamma)}$ is immaterial —only the bound $\left\|\left(A^{\prime}\right)^{-1}\right\|_{H^{s_{A}}(\Gamma) \leftarrow H^{s_{A}(\Gamma)}}$ appears in the estimates. With Lemma 5.7 and Remark 5.8 we write

$$
A^{\prime}= \begin{cases}-\frac{1}{2}+K_{0}^{\prime}+R_{A^{\prime}}+k\left[\widetilde{\mathcal{A}}_{A^{\prime}, 1}\right]+\left[\partial_{n} \widetilde{\mathcal{A}}_{A^{\prime}, 2}\right] & \text { if } A^{\prime}=-1 / 2+K^{\prime}+\mathbf{i} \eta V \\ \frac{1}{2}+K_{0}^{\prime}+\mathbf{i} V_{0}+R_{A^{\prime}}+k\left[\widetilde{\mathcal{A}}_{A^{\prime}, 1}\right]+\left[\partial_{n} \widetilde{\mathcal{A}}_{A^{\prime}, 2}\right] & \text { if } A^{\prime}=1 / 2+K^{\prime}+\mathbf{i} \eta V .\end{cases}
$$


This has the form required in Lemma 6.10, if we set

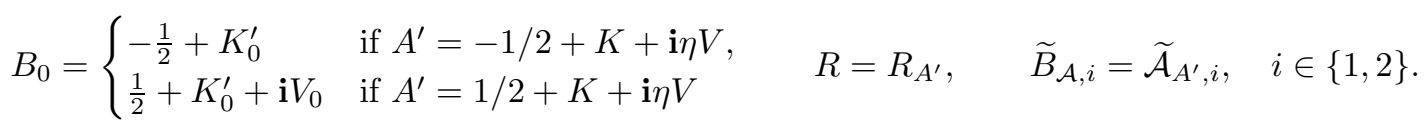

By Lemma 2.5, the operator $B_{0}$ is invertible on $H^{s}(\Gamma)$. Hence, selecting $q$ in Lemma 5.7 appropriately, we may assume $\left\|R B_{0}^{-1}\right\|_{H^{s}(\Gamma) \leftarrow H^{s}(\Gamma)}<1$. Lemma 5.7 provides the necessary information about the mapping properties of $\widetilde{B}_{\mathcal{A}, i}, i \in\{1,2\}$. Since $s \geq 0$, we conclude that (in the notation of Lemma 6.10) $C_{\text {smooth }}(k) \sim k$. From Corollary 6.9 we obtain

$$
C_{\text {solve }}(k) \sim k^{-1}\left(1+k^{5 / 2+s_{A}^{+}}\left\|\left(A^{\prime}\right)^{-1}\right\|_{\left.H^{s_{A} \leftarrow H^{s_{A}}}\right)} .\right.
$$

Lemma 6.10 then implies the result.

Theorem 6.12 restricts its attention to the case $s \geq 0$. However, the case $s=-1 / 2$ is particular interest given that it is the energy space for the operator $K^{\prime}$. We therefore modify the arguments slightly to cover this case as well:

Theorem 6.13 (decomposition of $\left(A^{\prime}\right)^{-1}$, negative norms). Let $\Gamma$ be analytic, $s_{A} \geq$ $-1 / 2,-1 \leq s \leq 0$. Let $A^{\prime}$ be boundedly invertible on $H^{\min \left\{s, s_{A}\right\}}(\Gamma)$. Then the operator $\left(A^{\prime}\right)^{-1}$ can be written as

$$
\left(A^{\prime}\right)^{-1}=A_{Z}^{\prime}+\gamma_{1}^{e x t} \widetilde{A}_{A^{\prime}}-\gamma_{1}^{i n t} \widetilde{A}_{A^{\prime}}
$$

where the linear operators $A_{A}^{\prime}$ and $\widetilde{A}_{A^{\prime}}$ satisfy with $s_{A}^{+}:=\max \left\{s_{A}, 0\right\}$

$$
\begin{aligned}
& \left\|A_{A}^{\prime}\right\|_{H^{s}(\Gamma) \leftarrow H^{s}(\Gamma)} \leq C, \\
& \widetilde{A}_{A^{\prime}} f \in \mathfrak{A}\left(C C_{f}, \gamma, T\right), \quad C_{f}:=k^{d / 2}\left(1+k^{5 / 2+s_{A}^{+}}\left\|\left(A^{\prime}\right)^{-1}\right\|_{\left.H^{s_{A} \leftarrow H^{s_{A}}}\right)}\right)\|f\|_{H^{s}(\Gamma)} .
\end{aligned}
$$

Here, $C, \gamma>0$, and the tubular neighborhood $T$ of $\Gamma$ are independent of $k \geq k_{0}$.

Proof. We proceed as in the proof of Theorem 6.12 but replace the decomposition of Lemma 5.7 with that of Lemma 5.9. That lemma leads to $C_{\text {smooth }}(k) \leq k^{d / 2}+k \sim$ $k^{d / 2}$. Since $C_{\text {solve }}(k)$ is given by $(6.15)$ we get the desired result.

Appendix A. Proofs of Lemmata 2.1, 2.2. Proof of Lemma 2.1: The result for $-1 / 2<s<1 / 2$ being known in the literature (see, e.g., [18]), we restrict our attention to the limiting cases $s= \pm 1 / 2$. We start with the case $s=1 / 2$. Set $u:=\widetilde{V}_{0} \varphi$ for $\varphi \in L^{2}(\Gamma)$. Then $u \in H^{3 / 2}\left(\Omega_{R}\right)$ with $\|u\|_{H^{3 / 2}\left(\Omega_{R}\right)} \leq C\|\varphi\|_{L^{2}(\Gamma)}$, which can be seen as follows: By [29, Thms. 3.3, 4.11] we have $\left\|V_{0} \varphi\right\|_{H^{1}(\Gamma)} \leq C\|\varphi\|_{L^{2}(\Gamma)}$. Since $\gamma_{0}^{\text {int } / \text { ext }} \widetilde{V}_{0} \varphi=V_{0} \varphi$, the uniqueness assertion of [14, Thm. 5.15] implies that $u=\widetilde{V}_{0} \varphi \in H^{3 / 2}\left(\Omega_{R}\right)$. Next, [14, Thm. 5.6, Cor. 5.7] imply

$$
\|u\|_{H^{3 / 2}\left(\Omega_{R}\right)}+\left\|\sqrt{\delta} \nabla^{2} u\right\|_{L^{2}\left(\Omega_{R}\right)}+\left\|u^{*}\right\|_{L^{2}(\Gamma)}+\left\|(\nabla u)^{*}\right\|_{L^{2}(\Gamma)} \leq C\|\varphi\|_{L^{2}(\Gamma)} ;
$$

here, the notation $v^{*}$ denotes the non-tangential maximal functions (see [29]) and $\delta(x)=\operatorname{dist}(x, \Gamma)$ denotes the distance from $\Gamma$.

Additionally, we have from [14, Prop. 2.18] that $u \in B_{2, \infty}^{3 / 2}\left(B_{R}\right)$ if and only if $u \in$ $L^{2}\left(B_{R}\right)$ and $\nabla u \in B_{2, \infty}^{1 / 2}\left(B_{R}\right)$. It therefore remains to assert $\nabla u \in B_{2, \infty}^{1 / 2}\left(B_{R}\right)$. To that end, consider $v=\partial_{i} u$ for a fixed $i$ and let $v_{\varepsilon}:=v \star \rho_{\varepsilon}$ be its regularization, where 
$\rho_{\varepsilon}$ is a standard mollifier with length scale $\varepsilon$. We have by standard arguments for each fixed $x \in B_{R}$ such that $v \in H^{1}\left(B_{2 \varepsilon}(x)\right)$ :

$$
\begin{aligned}
\left\|v-v_{\varepsilon}\right\|_{L^{2}\left(B_{\varepsilon}(x)\right)} & \leq \varepsilon\|\nabla v\|_{L^{2}\left(B_{2 \varepsilon}(x)\right)}, \\
\left\|\nabla v_{\varepsilon}\right\|_{L^{2}\left(B_{\varepsilon}(x)\right)} & \leq\|\nabla v\|_{L^{2}\left(B_{2 \varepsilon}(x)\right)} .
\end{aligned}
$$

For $\varepsilon>0$ we denote by $S_{\varepsilon}:=\cup_{x \in \Gamma} B_{\varepsilon}(x)$ the tubular neighborhood of $\Gamma$ of width $\varepsilon$. Covering the set $B_{R} \backslash S_{3 \varepsilon} \subset \cup_{x \in B_{R} \backslash S_{3 \varepsilon}} B_{\varepsilon}(x)$ we infer with the aid of Besicovitch's covering theorem

$$
\begin{aligned}
\left\|v-v_{\varepsilon}\right\|_{L^{2}\left(B_{R} \backslash S_{3 \varepsilon}\right)} & \leq C \varepsilon\|\nabla v\|_{L^{2}\left(B_{R} \backslash S_{\varepsilon}\right)} \leq \varepsilon^{1 / 2}\left\|\delta^{1 / 2} \nabla^{2} u\right\|_{L^{2}\left(\Omega_{R}\right)} \leq \varepsilon^{1 / 2}\|\varphi\|_{L^{2}(\Gamma)}, \\
\left\|\nabla v_{\varepsilon}\right\|_{L^{2}\left(B_{R} \backslash S_{3 \varepsilon}\right)} & \leq C\|\nabla v\|_{L^{2}\left(B_{R} \backslash S_{\varepsilon}\right)} \leq C \varepsilon^{-1 / 2}\left\|\delta^{1 / 2} \nabla v\right\|_{L^{2}\left(\Omega_{R}\right)} \leq C \varepsilon^{-1 / 2}\|\varphi\|_{L^{2}(\Gamma)} .
\end{aligned}
$$

For the regularized function $v_{\varepsilon}$ we have with the definition of the non-tangential maximal function and (A.1)

$$
\left\|v_{\varepsilon}\right\|_{L^{2}\left(S_{\varepsilon}\right)} \leq C\|v\|_{L^{2}\left(S_{2 \varepsilon}\right)} \leq C \varepsilon^{1 / 2}\left\|v^{*}\right\|_{L^{2}(\Gamma)} \leq C \varepsilon^{1 / 2}\|\varphi\|_{L^{2}(\Gamma)} .
$$

Finally, for the derivative we compute

$$
\left\|\nabla v_{\varepsilon}\right\|_{L^{2}\left(S_{3 \varepsilon}\right)} \leq C \varepsilon^{-1}\|v\|_{L^{2}\left(S_{4 \varepsilon}\right)} \leq C \varepsilon^{-1 / 2}\left\|v^{*}\right\|_{L^{2}(\Gamma)} \leq C \varepsilon^{-1 / 2}\|\varphi\|_{L^{2}(\Gamma)} .
$$

Thus, we obtain the following estimate for the $K$-functional:

$$
K(v, \varepsilon) \leq\left\|v-v_{\varepsilon}\right\|_{L^{2}\left(B_{R}\right)}+\varepsilon\left\|v_{\varepsilon}\right\|_{H^{1}\left(B_{R}\right)} \leq C \varepsilon^{1 / 2}\|\varphi\|_{L^{2}(\Gamma)} .
$$

Since $\varepsilon>0$ is arbitrary, we conclude $v \in B_{2, \infty}^{1 / 2}\left(B_{R}\right)$.

For the case $s=-1 / 2$ we start by noting that $V_{0}: H^{-1}(\Gamma) \rightarrow L^{2}(\Gamma)$, which follows from the self-adjointness of $V_{0}$, the above cite result by Verchota that $V_{0}: L^{2}(\Gamma) \rightarrow$ $H^{1}(\Gamma)$, and a duality argument. Next, we approximate $\varphi \in H^{-1}(\Gamma)$ by functions $\left(\varphi_{n}\right)_{n \in \mathbb{N}} \subset L^{2}(\Gamma)$. As above, [14, Thm. 5.15] implies that the functions $\widetilde{V}_{0} \varphi_{n}$ are the unique harmonic functions with Dirichlet data $V_{0} \varphi_{n}$. Combining an estimate due to Dahlberg (see [14, Thm. 5.3]) and [14, Cor. 5.5] implies that $\widetilde{V}_{0} \varphi_{n} \in H^{1 / 2}\left(\Omega_{R}\right)$ together with

$$
\left\|\widetilde{V}_{0} \varphi_{n}\right\|_{H^{1 / 2}\left(\Omega_{R}\right)} \leq C\left\|V_{0} \varphi_{n}\right\|_{L^{2}(\Gamma)} \leq C\left\|\varphi_{n}\right\|_{H^{-1}(\Gamma)} .
$$

By linearity of $\widetilde{V}_{0}$, the sequence $\left(\widetilde{V}_{0} \varphi_{n}\right)_{n}$ is a Cauchy sequence in $H^{1 / 2}\left(\Omega_{R}\right)$. Furthermore, it converges pointwise to $\widetilde{V}_{0} \varphi$. We conclude that $\widetilde{V}_{0} \varphi \in H^{1 / 2}\left(\Omega_{R}\right)$ and $\left\|\widetilde{V}_{0} \varphi\right\|_{H^{1 / 2}\left(\Omega_{R}\right)} \leq C\|\varphi\|_{H^{-1}(\Gamma)}$. Appealing once more to [14, Cor. 5.5], we get for $u:=\widetilde{V}_{0} \varphi$ that $\left\|u^{*}\right\|_{L^{2}(\Gamma)}+\|u\|_{H^{1 / 2}\left(\Omega_{R}\right)} \leq C\|\varphi\|_{H^{-1}(\Gamma)}$. Using now the same arguments as in the case $s=1 / 2$, we conclude $\|u\|_{B_{2, \infty}^{1 / 2}\left(B_{R}\right)} \leq C\|\varphi\|_{H^{-1}(\Gamma)}$.

The remaining cases $-1 / 2<s<1 / 2$ can now be inferred from the limiting cases $s= \pm 1 / 2$ by an interpolation argument.

Proof of Lemma 2.2: The proof is very similar to that of Lemma 2.1. The case $s=1 / 2$ is see as follows: For $\varphi \in H^{1}(\Gamma) \subset H^{1 / 2}(\Gamma)$, we have $\widetilde{K}_{0} \varphi \in H^{1}\left(\Omega_{R}\right)$. We have $\gamma_{0}^{\text {int } / \text { ext }} \widetilde{K}_{0} \varphi=\left(\mp 1 / 2+K_{0}\right) \varphi \in H^{1 / 2}(\Gamma) \subset L^{2}(\Gamma)$. By [14, Cor. 5.5], the interior and exterior non-tangential limits $\operatorname{Tr}^{i n t / e x t} \widetilde{K}_{0} \varphi$ on $\Gamma$ exist and are in $L^{2}(\Gamma)$. These must coincide with the interior and exterior traces $\gamma_{0}^{i n t / e x t} \widetilde{K}_{0} \varphi$ and we conclude 
$\operatorname{Tr}^{\text {int } / e x t} \widetilde{K}_{0} \varphi=\gamma_{0}^{\text {int } / e x t} \widetilde{K}_{0} \varphi=\left(\mp 1 / 2+K_{0}\right) \varphi$. By [29, Thm. 3.3] we have $(\mp 1 / 2+$ $\left.K_{0}\right) \varphi \in H^{1}(\partial \Omega)$, so that [14, Thm. 5.15] implies $\widetilde{K}_{0} \varphi \in H^{3 / 2}\left(\Omega_{R}\right)$. Then [14, Cor. 5.7] implies $\widetilde{K}_{0} \varphi \in H^{3 / 2}\left(\Omega_{R}\right)$ with $\left\|\widetilde{K}_{0} \varphi\right\|_{H^{3 / 2}\left(\Omega_{R}\right)} \leq C\|\varphi\|_{H^{1}(\Gamma)}$.

For the case $s=-1 / 2$, we proceed as in the proof of Lemma 2.1. First, we show for $\varphi \in L^{2}(\Gamma)$ that

$$
\left\|\widetilde{K}_{0} \varphi\right\|_{H^{1 / 2}\left(\Omega_{R}\right)}+\left\|\left(\widetilde{K}_{0} \varphi\right)^{*}\right\|_{L^{2}(\Gamma)} \leq C\|\varphi\|_{L^{2}(\Gamma)} .
$$

The assertion $\widetilde{K}_{0} \varphi \in B_{2, \infty}^{1 / 2}\left(B_{R}\right)$ follows from this in the same way as in the proof of Lemma 2.1. Finally, for $-1 / 2<s<1 / 2$ the assertion $\widetilde{K}_{0}: H^{1 / 2+s}(\Gamma) \rightarrow H^{1+s}\left(\Omega_{R}\right)$ follows by an interpolation argument from $\widetilde{K}_{0}: H^{1 / 2+s}(\Gamma) \rightarrow H^{1+s}\left(\Omega_{R}\right)$ for the limiting cases $s= \pm 1 / 2$, which have just been proved.

\section{Appendix B. regularity assertions for parameter-dependent elliptic PDEs.}

B.1. analytic regularity. We start with a lemma that shows that membership in the class $\mathfrak{A}$ of analytic functions is preserved under analytic changes of variables: Lemma B.1. Let $G, G_{1} \subset \mathbb{R}^{d}$ be bounded open sets. Assume that $g: \overline{G_{1}} \rightarrow \mathbb{R}^{d}$ is analytic, $\left|\operatorname{det} g^{\prime}\right|>0$ on $\overline{G_{1}}$ and that $g\left(G_{1}\right) \subset G$. Let $f_{1}: \overline{G_{1}} \rightarrow \mathbb{C}, f_{2}: \bar{G} \rightarrow \mathbb{C}$ be analytic and assume that $f_{2} \in \mathfrak{A}\left(C_{f}, \gamma_{f}, G\right)$. Then the function $F: x \mapsto f_{1}(x)\left(f_{2} \circ\right.$ $g)(x)$ satisfies $F \in \mathfrak{A}\left(C C_{f}, \gamma^{\prime}, G\right)$ for some constants $C$, $\gamma^{\prime}$ that depend solely on $\gamma$, $f_{1}, g$, and $k_{0}$.

Proof. The case $d=2$ is taken directly from [19, Lemma 4.3.1]. Inspection of the proof of [19, Lemma 4.3.1] shows that it can be generalized to $d>2$.

Next, we recall that if a function $u$ satisfies the differential equation

$$
-\nabla \cdot(B \nabla u)+k^{2} c u=f
$$

and if the function $F$ provides a sufficiently smooth change of variables, then the transformed function $\widehat{u}:=u \circ F$ solves

$$
-\nabla \cdot(\widehat{B} \nabla \widehat{u})+k^{2} \operatorname{det} F^{\prime} \widehat{c} \widehat{u}=\operatorname{det} F^{\prime} \widehat{f},
$$

where $\widehat{B}=B \circ F, \widehat{c}=c \circ F$, and $\widehat{f}=f \circ F$. Finally, for the convenience of referring to the assumptions on the coefficients $B$, $c$, we make the following assumptions: The matrix-valued function $B$ is pointwise symmetric positive definite and

$$
\begin{aligned}
& 0<\lambda_{\min }<B(x) \quad \forall x \in \omega, \\
& \left\|\nabla^{n} c\right\|_{L^{\infty}(\omega)} \leq C_{c} \gamma_{c}^{n} n !, \quad\left\|\nabla^{n} B\right\|_{L^{\infty}(\omega)} \leq C_{B} \gamma_{B}^{n} n ! \quad \forall n \in \mathbb{N}_{0} .
\end{aligned}
$$

Theorem B.2 (Dirichlet b.c.). Let $\omega \subset \mathbb{R}^{d}$ be a bounded Lipschitz domain with analytic boundary. Assume (B.2). Let $u \in H^{1}(\omega)$ solve (B.1) on $\omega$ for an $f \in$ $\mathfrak{A}\left(C_{f}, \gamma_{f}, \omega\right)$. Assume that $u$ satisfies $\left.u\right|_{\partial \omega}=\left.G\right|_{\partial \omega}$ for a $G \in \mathfrak{A}\left(C_{G}, \gamma_{G}, \omega \cap T^{\prime}\right)$, where $T^{\prime}$ is a tubular neighborhood of $\partial \omega$. Fix a tubular neighborhood $T$ of $\partial \omega$ with $\bar{T} \subset T^{\prime}$. Then u satisfies

$$
u \in \mathfrak{A}\left(C C_{u}, \gamma_{u}, \omega \cap T\right), \quad C_{u}:=k^{-2} C_{f}+C_{G}+k^{-1}\|u\|_{\mathcal{H}, T^{\prime} \cap \omega} .
$$

where the constants $C$ and $\gamma_{u}$ depend solely on $\gamma_{G}, \gamma_{f}, \partial \omega, k_{0}$, and the constants of (B.2). 
Proof. Consider the function $z:=u-G$. Since $G \in \mathfrak{A}\left(C_{G}, \gamma_{G}, \omega \cap T^{\prime}\right)$, it suffices to establish $z \in \mathfrak{A}\left(C C_{u}, \gamma_{u}, \omega \cap T\right)$. The function $z$ satisfies

$-k^{-2} \nabla \cdot(B \nabla z)-c z=\tilde{f}:=k^{-2} f-k^{-2} \nabla \cdot(B \nabla G)-c G \quad$ on $T^{\prime} \cap \omega,\left.\quad z\right|_{\partial \omega}=0$.

The assumptions on $f$ and $G$ and Lemma B.1 imply $\widetilde{f} \in \mathfrak{A}\left(C\left(k^{-2} C_{f}+C_{G}\right), \widetilde{\gamma}, T^{\prime} \cap \omega\right)$ for some constants $C, \widetilde{\gamma}$. From [19, Props. 5.5.1, 5.5.2] we get $z \in \mathfrak{A}\left(C\left(k^{-2} C_{f}+C_{G}+\right.\right.$ $\left.\left.k^{-1}\|z\|_{\mathcal{H}, T^{\prime} \cap \omega}\right), \gamma, T \cap \omega\right)$. Since $k^{-1}\|z\|_{\mathcal{H}, T^{\prime} \cap \omega} \leq C\left(C_{G}+k^{-1}\|u\|_{\mathcal{H}, T^{\prime} \cap \omega}\right)$, the desired result now follows. $\square$

Theorem B.3 (Robin b.c.). Let $\omega \subset \mathbb{R}^{d}$ be a bounded Lipschitz domain with analytic boundary. Assume (B.2). Let $u \in H^{1}(\omega)$ solve (B.1) on $\omega$ for an $f \in \mathfrak{A}\left(C_{f}, \gamma_{f}, \omega\right)$. Assume that $u$ satisfies

$$
\gamma_{1}^{i n t} u=\gamma_{0}^{i n t} G_{1}+\mathbf{i} k\left(\gamma_{0}^{i n t} G_{2}\right) \gamma_{0}^{i n t} u
$$

where, for some tubular neighborhood $T^{\prime}$ of $\partial \omega$ we have $G_{1} \in \mathfrak{A}\left(C_{G_{1}}, \gamma_{G_{1}}, \omega \cap T^{\prime}\right)$ and $G_{2}$ is analytic on $T^{\prime}$. Here, the trace operators $\gamma_{0}^{\text {int }}$ and $\gamma_{1}^{\text {int }}$ are understood with respect to $\omega$. Fix a tubular neighborhood $T$ of $\partial \omega$ with $\bar{T} \subset T^{\prime}$. Then u satisfies

$$
u \in \mathfrak{A}\left(C C_{u}, \gamma_{u}, \omega \cap T\right), \quad C_{u}:=k^{-2} C_{f}+k^{-1} C_{G_{1}}+k^{-1}\|u\|_{\mathcal{H}, T^{\prime} \cap \omega},
$$

where $C$ and $\gamma_{u}$ depend solely on $\gamma_{G_{1}}, \gamma_{f}, \partial \omega, G_{2}, k_{0}$, and the constants of (B.2).

Proof. The proof is sketched for a related 2D problem in [19, Prop. 5.4.5, Rem. 5.4.6]. The key observation is again that Lemma B.1 allows us to locally flatten the boundary while preserving the structure of the differential equation and the boundary conditions. Then the technique employed in [19, Prop. 5.4.5] is applicable.

Theorem B.4 (transmission conditions). Let $\omega^{\prime}, \omega \subset \mathbb{R}^{d}$ be two bounded domains with $\omega^{\prime} \subset \subset \omega$. Denote $\gamma:=\partial \omega^{\prime}$ and assume that $\gamma$ is analytic. Assume (B.2). Let $u \in H^{1}(\omega)$ solve (B.1) on $\omega$ for an $f \in \mathfrak{A}\left(C_{f}, \gamma_{f}, \omega \backslash \gamma\right)$. Fix $\omega^{\prime \prime} \subset \subset \omega$. Then

$$
u \in \mathfrak{A}\left(C C_{u}, \gamma_{u}, \omega^{\prime \prime} \backslash \gamma\right), \quad C_{u}:=k^{-2} C_{f}+k^{-1}\|u\|_{\mathcal{H}, \omega}
$$

for some constants $C, \gamma_{u}>0$ that depend solely on $\gamma_{f}, \omega^{\prime}, \omega^{\prime \prime}, \omega, k_{0}$, and the constants of (B.2).

Proof. The interesting estimates are those near the boundary $\gamma$. Here, the standard procedure of locally flattening $\gamma$ can be brought to bear in view of Lemma B.1. Then, [19, Prop. 5.5.4] is applicable. $\square$

Lemma B.5. Let $\omega \subset \mathbb{R}^{d}$ be a bounded Lipschitz domain with analytic boundary $\partial \omega$. Set $\omega^{+}:=\mathbb{R}^{d} \backslash \bar{\omega}$. Let $T$ be a tubular neighborhood of $\partial \omega$. Let $G \in \mathfrak{A}\left(C_{G}, \gamma_{G}, T \cap \omega\right)$. Then there exists a tubular neighborhood $\widetilde{T}$ of $\partial \omega$ and constants $C, \gamma_{\widetilde{G}}$ that depend solely on $\gamma_{G}, \partial \omega, k_{0}$ with the following property: There exists a $\widetilde{G} \in \mathfrak{A}\left(C C_{G}, \gamma_{\widetilde{G}}, \widetilde{T} \cap\right.$ $\left.\omega^{+}\right)$with $\gamma_{0}^{\text {ext }} \widetilde{G}=\gamma_{0}^{\text {int }} G$. Here, $\gamma_{0}^{\text {ext }}$ and $\gamma_{0}^{\text {int }}$ are the trace operators with respect to $\omega$.

Proof. The idea is to define $\widetilde{G}$ by reflection at $\partial \omega$. One can define boundary fitted coordinates $\psi: \partial \omega \times(-\varepsilon, \varepsilon) \rightarrow \mathbb{R}^{d}$ via $\psi(x, \rho):=x+\rho \vec{n}(x)$, where $\vec{n}(x)$ is the (outer) normal vector of $\partial \omega$ at $x \in \partial \omega$. Since $\partial \omega$ is assumed to be analytic, $\psi$ is likewise analytic. For $\varepsilon>0$ sufficiently small, the range of $\psi$ is a tubular neighborhood (denoted $T$ ) of $\partial \omega$ and restricted to $T$, the inverse $\psi^{-1}$ of $\psi$ exists and is analytic. We write $\psi^{-1}(x)=(\gamma(x), \rho(x))$. For $x \in T \cap \omega^{+}$we then define $G^{+}(x)$ by $G^{+}(x):=$ $G(\psi(a(x),-\rho(x)))$. The analyticity of $\psi^{-1}$ and Lemma B.1 then implies the result. 
B.2. finite regularity. THEOREM B.6. Let $\omega^{\prime}$ and $\omega \subset \mathbb{R}^{d}$ be two bounded domains with $\omega^{\prime} \subset \subset \omega$. Denote by $\gamma:=\partial \omega^{\prime}$ and assume that $\gamma$ is analytic. Assume (B.2). Let $u \in H^{1}(\omega)$ solve (B.1) on $\omega$ for some $f \in H^{s}(\omega \backslash \gamma)$ with $s \geq 0$. Fix $\omega^{\prime \prime} \subset \subset \omega$. If $s \in \mathbb{N}_{0}$, then

$$
\sum_{n=0}^{s} k^{-(n+2)}\left\|\nabla^{n+2} u\right\|_{L^{2}\left(\omega^{\prime \prime} \backslash \gamma\right)} \leq C\left[\sum_{j=0}^{s} k^{-j-2}\left\|\nabla^{j} f\right\|_{L^{2}(\omega \backslash \gamma)}+\|u\|_{L^{2}(\omega)}\right]
$$

where the constant $C$ depends on $s$ but is independent of $k \geq k_{0}$ and $u$. If we assume $s \geq 0$, then for some $C>0$ independent of $k \geq k_{0}$ and $u$ :

$$
\|u\|_{H^{s+2}\left(\omega^{\prime \prime} \backslash \gamma\right)} \leq C\left[k^{s}\|f\|_{L^{2}(\omega)}+\|f\|_{H^{s}(\omega)}+k^{s+2}\|u\|_{L^{2}(\omega)}\right] .
$$

Proof. We start by observing that standard elliptic regularity (note that the interface $\gamma$ is smooth) for

$$
-\nabla \cdot(B \nabla \widetilde{u})=\widetilde{f} \quad \text { on } \omega
$$

gives for $s \geq 0$ and any domain $\widetilde{\omega}$ with $\omega^{\prime \prime} \subset \subset \widetilde{\omega} \subset \subset \omega$

$$
\|\widetilde{u}\|_{H^{s+2}\left(\omega^{\prime \prime} \backslash \gamma\right)} \lesssim\|\widetilde{f}\|_{H^{s}(\widetilde{\omega} \backslash \gamma)}+\|u\|_{L^{2}(\widetilde{\omega})} .
$$

We apply this result with $\tilde{f}=f+k^{2} c u$, multiply through with $k^{-s}$, and get

$$
k^{-s}\|u\|_{H^{s+2}\left(\omega^{\prime \prime} \backslash \gamma\right)} \lesssim k^{-s}\|f\|_{H^{s}(\widetilde{\omega} \backslash \gamma)}+k^{-(s-2)}\|u\|_{H^{s}(\widetilde{\omega} \backslash \gamma)}+k^{-s}\|u\|_{L^{2}(\widetilde{\omega})} .
$$

For even integer $s \in 2 \mathbb{N}_{0}$, we can iterate (B.5) to get

$$
k^{-s}\|u\|_{H^{s+2}\left(\omega^{\prime \prime} \backslash \gamma\right)} \lesssim \sum_{j=0}^{s / 2} k^{-2 j}\|f\|_{H^{2 j}(\omega \backslash \gamma)}+k^{2}\|u\|_{L^{2}(\omega \backslash \gamma)}, \quad s \in 2 \mathbb{N}_{0} .
$$

For odd $s \in 1+2 \mathbb{N}_{0}$ we get analogously

$$
k^{-s}\|u\|_{H^{s+2}\left(\omega^{\prime \prime} \backslash \gamma\right)} \lesssim \sum_{j=0}^{(s+1) / 2-1} k^{-2 j-1}\|f\|_{H^{2 j+1}(\omega \backslash \gamma)}+k\|u\|_{H^{1}(\omega \backslash \gamma)}+k^{-1}\|u\|_{L^{2}(\omega \backslash \gamma)}
$$

The bound (B.6) with $s=0$ produces $\|u\|_{H^{2}\left(\omega^{\prime \prime} \backslash \gamma\right)} \lesssim\|f\|_{L^{2}(\omega \backslash \gamma)}+k^{2}\|u\|_{L^{2}(\omega \backslash \gamma)}$. Combining this with the standard (piecewise) interpolation inequality

$$
\|u\|_{H^{1}(\omega \backslash \gamma)} \lesssim\|u\|_{H^{2}(\omega \backslash \gamma)}^{1 / 2}\|u\|_{L^{2}(\omega \backslash \gamma)}^{1 / 2} \lesssim k^{-1}\|u\|_{H^{2}(\omega \backslash \gamma)}+k\|u\|_{L^{2}(\omega \backslash \gamma)}
$$

and appropriately adjusting the domains, we can conclude for $s \in \mathbb{N}_{0}$

$$
k^{-s}\|u\|_{H^{s+2}\left(\omega^{\prime \prime} \backslash \gamma\right)} \lesssim \sum_{j=0}^{s} k^{-j}\|f\|_{H^{j}(\omega \backslash \gamma)}+k^{2}\|u\|_{L^{2}(\omega \backslash \gamma)}
$$

from which we derive (B.3). For the proof of (B.4) we introduce the notation $\sigma:=\lfloor s\rfloor$ and observe the (piecewise) interpolation inequality

$$
\|u\|_{H^{s}(\omega \backslash \gamma)} \lesssim\|u\|_{L^{2}(\omega \backslash \gamma)}^{1-\theta_{1}}\|u\|_{H^{\sigma+2}(\omega \backslash \gamma)}^{\theta_{1}}, \quad \theta_{1}:=\frac{s}{\sigma+2} .
$$


For every $\varepsilon_{1}>0$ we get from Young's inequality

$$
\|u\|_{H^{s}(\omega \backslash \gamma)} \lesssim \varepsilon_{1}^{1 /\left(1-\theta_{1}\right)}\|u\|_{L^{2}(\omega \backslash \gamma)}+\varepsilon_{1}^{-1 / \theta_{1}}\|u\|_{H^{\sigma+2}(\omega \backslash \gamma)} .
$$

Selecting $\varepsilon_{1}:=k^{s\left(1-\theta_{1}\right)}$ we arrive at

$$
\|u\|_{H^{s}(\omega \backslash \gamma)} \lesssim k^{s}\|u\|_{L^{2}(\omega \backslash \gamma)}+k^{s-\sigma-2}\|u\|_{H^{\sigma+2}(\omega \backslash \gamma)} .
$$

Next, we use again a (piecewise) interpolation inequality to bound for $0 \leq j \leq \sigma<s$ and Young's inequality

$$
k^{s-j}\|f\|_{H^{j}(\omega \backslash \gamma)} \lesssim\left(k^{s}\|f\|_{L^{2}(\omega \backslash \gamma)}\right)^{1-j / s}\|f\|_{H^{s}(\omega \backslash \gamma)}^{j / s} \lesssim k^{s}\|f\|_{L^{2}(\omega \backslash \gamma)}+\|f\|_{H^{s}(\omega \backslash \gamma)} .
$$

Combining (B.9), (B.8), (B.7) we arrive at the desired bound (B.4).

Appendix C. regularity of Laplace-Beltrami eigenfunctions. Let $\Omega \subset \mathbb{R}^{d}$ be a bounded domain with an analytic boundary $\Gamma$. Let $\left(\varphi_{m}, \lambda_{m}^{2}\right), m \in \mathbb{N}_{0}$, be the eigenpairs of the Laplace-Beltrami operator, i.e.,

$$
-\Delta_{\Gamma} \varphi_{m}=\lambda_{m}^{2} \varphi_{m} \quad \text { on } \Gamma .
$$

We assume that the eigenvalues $\lambda_{m} \geq 0$ are sorted in ascending order and that the eigenfunctions $\left(\varphi_{m}\right)_{m \in \mathbb{N}_{0}}$ are orthonormalized in $L^{2}(\Gamma)$.

Lemma C.1 (analytic regularity of $\varphi_{m}$ ). Let $\Gamma$ be analytic. Then there exist constants $C, \gamma>0$ independent of $m$ such that

$$
\left\|\nabla_{\Gamma}^{n} \varphi_{m}\right\|_{L^{2}(\Gamma)} \leq C \max \left\{\lambda_{m}, n\right\}^{n} \gamma^{n} \quad \forall n \in \mathbb{N}_{0}
$$

where $\nabla_{\Gamma}$ denotes the surface gradient. Furthermore, there exists a tubular neighborhood $T$ of $\Gamma$ (depending solely on $\Gamma$ ) such that all functions $\varphi_{m}$ can be extended to analytic functions (again denoted $\varphi_{m}$ ) on $T$ that satisfy

$$
\left\|\nabla^{n} \varphi_{m}\right\|_{L^{2}(T)} \leq C \max \left\{\lambda_{m}, n\right\}^{n} \gamma^{n} \quad \forall n \in \mathbb{N}_{0}
$$

Proof. Sketch of the proof: If $\gamma: U \rightarrow \Gamma$ for some $U \subset \mathbb{R}^{d-1}$ is one of the analytic charts, then the Laplace-Beltrami operator $\Delta_{\Gamma}$ applied to a function $u: \Gamma \rightarrow \mathbb{R}$ has the following form on $U$ :

$$
\frac{1}{\sqrt{g}} \sum_{i, j=1}^{d-1} \partial_{i}\left(\sqrt{g} g^{i j} \partial_{j}(u \circ \gamma)\right)
$$

where $g=\operatorname{det} G$ is the determinant of the metric tensor $G$ given by $G_{i j}:=\partial_{i} \gamma \cdot \partial_{j} \gamma$ and the matrix $\left(g^{i j}\right)_{i, j=1}^{d}$ is the (pointwise) inverse of $G$. The matrix $G$ is pointwise symmetric positive definite and thus also its inverse $\left(g^{i j}\right)_{i, j=1}^{d}$. By the analyticity of the charts, the matrices $\left(g^{i j}\right)_{i, j=1}^{d}$ and the function $g$ are analytic. On $U$, the pull-back $\widehat{\varphi}_{m}:=\varphi \circ \gamma$ of the eigenfunction $\varphi_{m}$ satisfies for the analytic, pointwise symmetric positive definite matrix $A_{i j}=\sqrt{g} g^{i j}$

$$
-\lambda_{m}^{-2} \nabla \cdot\left(A \nabla \widehat{\varphi}_{m}\right)-\sqrt{g} \widehat{\varphi}_{m}=0,
$$

Fix $K \subset \subset K^{\prime} \subset \subset U$. Then [19, Prop. 5.5.1] gives

$$
\left\|\nabla^{n+2} \widehat{\varphi}_{m}\right\|_{L^{2}(K)} \leq \max \left\{n, \lambda_{m}\right\}^{n+2} \gamma^{n}\left(\lambda_{m}^{-1}\left\|\nabla \widehat{\varphi}_{m}\right\|_{L^{2}\left(K^{\prime}\right)}+\lambda_{m}^{-2}\left\|\widehat{\varphi}_{m}\right\|_{L^{2}\left(K^{\prime}\right)}\right) \text {. }
$$


We have $\left\|\varphi_{m}\right\|_{H^{1}(\Gamma)} \sim \lambda_{m}$, and $\left\|\varphi_{m}\right\|_{L^{2}(\Gamma)}=1$. Hence,

$$
\left\|\widehat{\varphi}_{m}\right\|_{L^{2}\left(K^{\prime}\right)} \leq C, \quad\left\|\widehat{\varphi}_{m}\right\|_{H^{1}\left(K^{\prime}\right)} \leq C \lambda_{m} .
$$

Combining (C.3), (C.4) we see that

$$
\left\|\nabla^{n+2} \widehat{\varphi}_{m}\right\|_{L^{2}(K)} \leq C \max \left\{n, \lambda_{m}\right\}^{n+2} \gamma^{n} \quad \forall n \in \mathbb{N}_{0} \cup\{-1,-2\} .
$$

Returning to $\Gamma$ gives (C.1) in view of Lemma B.1. To see (C.2), we define the extension of $\varphi_{m}$ in the trivial way: In a tubular neighborhood $T$ of $\Gamma$ one can define boundary fitted coordinates $\Gamma \times[-\varepsilon, \varepsilon] \rightarrow T$ via $(x, \rho) \mapsto x+\rho \vec{n}(x)$, where $\vec{n}(x)$ is the (outer) normal vector at $x \in \Gamma$. For sufficiently small $\varepsilon$, this is a bijection, and we can define the extension by $\varphi_{m}(x+\rho \vec{n}(x))=\varphi_{m}(x)$.

REMARK C.2. Taking the trivial extension to the tubular neighborhood $T$ is clearly not the only choice. For example, if one is only interested in extending $\varphi_{m}$ only to $\Omega^{+} \cap T$ then one can select the extension to be $\varphi_{m}(x+\rho \vec{n}(x))=\varphi_{m}(x) e^{-\rho / \max \left\{\lambda_{m}, k\right\}}$, leading to slightly improved bounds in (C.2).

\section{REFERENCES}

[1] R. A. Adams. Sobolev Spaces. Academic Press, 1975.

[2] J. Bergh and J. Löfström. Interpolation Spaces. Springer Verlag, 1976.

[3] Helmut Brakhage and Peter Werner. Über das Dirichletsche Aussenraumproblem für die Helmholtzsche Schwingungsgleichung. Arch. Math., 16:325-329, 1965.

[4] S.N. Chandler-Wilde, I.G. Graham, S. Langdon, and M. Lindner. Condition number estimates for combined potential boundary integral operators in acoustic scattering. Journal of Integral Equations and Applications, 21:229-279, 2009.

[5] S.N. Chandler-Wilde and S. Langdon. A wavenumber independent BEM for an acoustic scattering problem. SIAM J. Numer. Anal., 46:2450-2477, 2006.

[6] S.N. Chandler-Wilde and S. Langdon. A Galerkin BEM for high frequency scattering by convex polygons. SIAM Journal on Numerical Analysis, 43:610-640, 2007.

[7] S.N. Chandler-Wilde and P. Monk. Wave-number-explicit bounds in time-harmonic scattering. SIAM J. Math. Anal, 39:1428-1455, 2008.

[8] I. Chavel. Eigenvalues in Riemannian Geometry. Academic Press, 1984.

[9] David L. Colton and Rainer Kress. Integral equation methods in scattering theory. Pure and Applied Mathematics (New York). John Wiley \& Sons Inc., New York, 1983.

[10] M. Costabel. Boundary integral operators on Lipschitz domains: elementary results. SIAM J. Math. Anal, 19(3):613-625, 1988.

[11] V. Domínguez, I. Graham, and V. Smyshlyaev. A hybrid numerical-asymptotic boundary integral method for high-frequency acoustic scattering. Numer. Math., 106:471-510, 2007.

[12] George C. Hsiao and Wolfgang L. Wendland. Boundary integral equations, volume 164 of Applied Mathematical Sciences. Springer-Verlag, Berlin, 2008.

[13] D.S. Jerison and C.E. Kenig. Boundary value problems on Lipschitz domains. In W. Littman, editor, Studies in Partial Differential Equations, volume 23, pages 1-68. MAA Studies in Math., 1982.

[14] D.S. Jerison and C.E. Kenig. The inhomogeneous Dirichlet problem in Lipschitz domains. J. Funct. Anal., 130:161-219, 1995.

[15] Rolf Leis. Zur Dirichletschen Randwertaufgabe des Aussenraumes der Schwingungsgleichung. Math. Z., 90:205-211, 1965.

[16] M. Löhndorf and J.M. Melenk. Mapping properties of Helmholtz boundary integral operators and their application to the $h p$-BEM. Technical Report 34/2009, Institute for Analysis and Scientific Computing, TU Wien, 2009.

[17] M. Löhndorf and J.M. Melenk. Wavenumber-explicit $h p$-BEM for high frequency scattering. Technical Report 02/2010, Institute for Analysis and Scientific Computing, TU Wien, 2010.

[18] W. McLean. Strongly elliptic systems and boundary integral equations. Cambridge University Press, 2000.

[19] J.M. Melenk. hp finite element methods for singular perturbations, volume 1796 of Lecture Notes in Mathematics. Springer Verlag, 2002. 
[20] J.M. Melenk. On approximation in meshless methods. In J. Blowey and A. Craig, editors, Frontier in Numerical Analysis, Durham 2004. Springer Verlag, 2005.

[21] J.M. Melenk and S.A. Sauter. Wave-number explicit convergence analysis for finite element discretizations of the Helmholtz equation (extended version). Technical Report 31/2009, Inst. for Analysis and Sci. Computing, Vienna Univ. of Technology, 2009.

[22] J.M. Melenk and S.A. Sauter. Convergence analysis for finite element discretizations of the Helmholtz equation with Dirichlet-to-Neumann boundary conditions. Math. Comp., (in press). available at http://www.math.tuwien.ac.at/ ${ }^{\sim}$ melenk/publications.

[23] O. I. Panič. On the solubility of exterior boundary-value problems for the wave equation and for a system of Maxwell's equations. Uspehi Mat. Nauk, 20(1 (121)):221-226, 1965.

[24] S. Sauter and C. Schwab. Randelementmethoden. Teubner, 2004.

[25] E.M. Stein. Singular integrals and differentiability properties of functions. Princeton University Press, 1970.

[26] O. Steinbach. Numerical Approximation Methods for Elliptic Boundary Value Problems: Finite and Boundary Elements. Springer Verlag, 2008.

[27] Luc Tartar. An introduction to Sobolev spaces and interpolation spaces, volume 3 of Lecture Notes of the Unione Matematica Italiana. Springer, Berlin, 2007.

[28] H. Triebel. Interpolation Theory, Function Spaces, Differential Operators. Johann Ambrosius Barth, 2 edition, 1995.

[29] G.C. Verchota. Layer potentials and regularity for the Dirichlet problem for Laplace's equation in Lipschitz domains. J. Funct. Anal., 59:572-611, 1984. 